\title{
A STUDY OF CERTAIN FUNCTIONAL EQUATIONS FOR THE $\vartheta$-FUNCTIONS*
}

BY

\section{E. B. VAN VLECK AND F. H'DOUBLER}

Functional equations for familiar elementary functions have been studied by Cauchy and others. In the case of the sine and cosine it is natural to treat the two functions together and to take as the defining system the addition-equations,

$$
\begin{aligned}
& S(x+y)=S(x) C(y)+C(x) S(y), \\
& C(x+y)=C(x) C(y)-S(x) S(y) .
\end{aligned}
$$

This system of equations has been studied by Tannery $\dagger$ and in Osgood's Lehrbuch der Funktionentheorie, $\ddagger$ and the real solutions are shown to have the form

$$
S(x)=e^{a x} \sin b x, \quad C(x)=e^{a x} \cos b x,
$$

from which the trigonometric functions are separated by imposing the accessory condition

$$
S^{2}(x)+C^{2}(x)=1 .
$$

The general solution of $(D)$ is given incidentally in this paper $(\S 7)$ on the hypothesis that $S(x), C(x)$ are continuous functions (not necessarily analytic) existent over the entire complex $x$-plane.

So far as we know, no previous investigation has been made of the more comprehensive system

$$
\begin{aligned}
& \phi(x+y) \phi(x-y)=\phi^{2}(x) \psi^{2}(y)-\phi^{2}(y) \psi^{2}(x), \\
& \psi(x+y) \psi(x-y)=\psi^{2}(x) \psi^{2}(y)-\phi^{2}(x) \phi^{2}(y),
\end{aligned}
$$

which forms the object of study of this paper. Uur purpose is two-fold, to ascertain what solutions this system admits and, secondly, when $\phi(x)$, $\psi(x)$ possess doubly periodic systems of zeros, to develop directly from $(A)$ the characteristic properties found for them in the theory of the Jacobian

* Presented to the Society at its summer meeting in Madison, September 9, 1913.

$\dagger$ Fonctions d'une variable, 1886, p. 147.

† See second edition, p. 582 . 
$\vartheta$-functions or the Weierstrassian $\sigma$-functions. In connection with $(A)$ the functional equation

$$
R(x+y) R(x-y)=\frac{R^{2}(x)-R^{2}(y)}{1-R^{2}(x) R^{2}(y)}
$$

is also treated.

It will be supposed that $\phi(x), \psi(x)$ are continuous one-valued functions (not necessarily analytic) existent over the entire plane, except in the investigation of real solutions of $(A)$ when existence is only assumed for the real axis. In any solution the components $\phi(x), \psi(x)$ can be modified by a common arbitrary exponential factor of form (11).

The different kinds of solutions can be differentiated from one another by the manner in which the roots are distributed. If $\phi(x) \equiv 0$, we have the equation

$$
\psi(x+y) \psi(x-y)=\psi^{2}(x) \psi^{2}(y),
$$

of which the complete solution is obtained $(\$ 2)$. A second case is that in which $\phi(x)$ vanishes identically along a straight line through the origin, or over a set of equidistant parallel lines. This case is discharged in $\S 3$. The solutions are non-analytic and are deducible in most simple fashion from sections of solutions for the other cases taken along the real axis or other line through the origin.

The only other possible cases are those in which the zeros of $\phi(x)$ and those of $\psi(x)$ form simultaneously either simply periodic or doubly periodic systems. A necessary and sufficient condition for the simply periodic case is obtained, depending upon the value of $\phi(x) / \phi(\alpha / 2)$ for $x=\alpha / 4$, where $\alpha$ designates a "primitive" zero used in normalizing $\phi(x)$. The complete solution $(\$ 7)$ is obtained for this case, the analytic solutions being expressible through the functions

$$
\phi(x)=e^{c x^{2}} \sin \mu x, \quad \psi(x)=e^{c x^{2}} \cos \mu x,
$$

or through "equivalent" $(\$ 1)$ solutions. All non-analytic solutions of this case are obtainable from these by a real affine transformation of the $x$-plane which leaves the origin unaltered. It may be added that by simple transformations equations $(A)$ may be reduced in this simply periodic case to form $(D)$.

In the remaining case of a doubly periodic system of zeros the existence of three "allied solutions" $(\$ 6)$ is established which together involve four functions having the characteristic properties of the four $\vartheta$-functions. When analytic, the solution is expressible through

$$
\phi(x)=\frac{\vartheta_{1}(c x, q)}{\vartheta(0, q)} e^{c x^{2}}, \quad \psi(x)=\frac{\vartheta(c x, q)}{\vartheta(0, q)} e^{c / x^{2}},
$$

and equivalent solutions, or through other similar pairs of $\vartheta$-functions (cf. 
I-III). It seems altogether likely that all non-analytic solutions are derivable from these by affine transformation of the $x$-plane, but the point is not settled.

All solutions are analytic on any line through the origin. A complete determination of the real solutions $(\S 10)$ is made, three cases (beside transitional cases) being distinguished which are solved through the functions:

$$
\begin{array}{ll}
\phi_{1}(x)=\frac{\vartheta_{1}(c x, q)}{\vartheta(0, q)} e^{c x^{2}}, & \psi(x)= \pm \frac{\vartheta(c x, q)}{\vartheta(0, q)} e^{c \prime x^{2}} \\
\phi_{2}(x)=\frac{\vartheta_{1}(c x, q)}{\vartheta_{2}(0, q)} e^{c / x^{2}}, & \psi(x)= \pm \frac{\vartheta_{2}(c x, q)}{\vartheta_{2}(0, q)} e^{c / x^{2}} \\
\phi_{3}(x)=\frac{\vartheta_{1}(c x, i q)}{\vartheta_{2}(0, i q)} e^{c \prime x^{2}}, & \psi(x)= \pm \frac{\vartheta_{2}(c x, i q)}{\vartheta_{2}(0, i q)} e^{c / x^{2}},
\end{array}
$$

in which $q, c, c^{\prime}$ are real and arbitrary with $|q|<1$. In terms of Weierstrassian functions we can give the solutions the more symmetrical form

$$
\begin{array}{r}
\phi(x)=e^{c / x^{2}} \sqrt{\left(e_{\alpha}-e_{\beta}\right)\left(e_{\alpha}-e_{\gamma}\right)} \sigma(c x), \quad \psi(x)= \pm e^{c / x^{2}} \sigma_{a}(c x) \\
(\alpha=1,2,3) .
\end{array}
$$

For $\alpha=1,3$ the quasi-periods of the functions are the one real and the other a pure imaginary, and we have the order of succession $e_{1}>e_{2}>e_{3}$. When $\alpha=2$, the periods are conjugate imaginaries, $e_{2}$ is real, and $e_{1}, e_{3}$ are conjugate imaginaries. In the transition case we get the solutions:

$$
\begin{array}{ll}
\phi(x)=e^{c x^{2}} \sinh \mu x, & \psi(x)= \pm e^{c x^{2}} \cosh \mu x, \\
\phi(x)=e^{c x^{2}} \sin \mu x, & \psi(x)= \pm e^{c x^{2}} \cos \mu x .
\end{array}
$$

It should be mentioned that it follows directly from $(A)$ that $\phi(x)$ satisfies the équation à trois termes:

$$
\begin{aligned}
& f\left(u_{1}+u_{2}\right) f\left(u_{1}-u_{2}\right) f\left(u_{3}+u_{4}\right) f\left(u_{3}-u_{4}\right) \\
& +f\left(u_{1}+u_{3}\right) f\left(u_{1}-u_{3}\right) f\left(u_{4}+u_{2}\right) f\left(u_{4}-u_{2}\right) \\
& \quad+f\left(u_{1}+u_{4}\right) f\left(u_{1}-u_{4}\right) f\left(u_{2}+u_{3}\right) f\left(u_{2}-u_{3}\right)=0 .
\end{aligned}
$$

The solution of this equation was obtained by Delisle* through power-series methods on the hypothesis of the analytic character of $f(u)$. With one assumption confessedly unproved regarding a certain determinant, the solution was shown to be of form $c^{\prime} e^{c x^{2}} \sigma(x)$.

\section{ON the zeros OF the solutions}

Let $\phi(x), \psi(x)$ be one-valued, continuous functions satisfying the equations

* Mathematis che Annalen, vol. 30 (1887), p. 91. 


$$
\left\{\begin{array}{l}
(1) \phi(x+y) \phi(x-y)=\phi^{2}(x) \psi^{2}(y)-\phi^{2}(y) \psi^{2}(x), \\
(2) \quad \psi(x+y) \psi(x-y)=\psi^{2}(x) \psi^{2}(y)-\phi^{2}(x) \phi^{2}(y) .
\end{array}\right.
$$

We shall suppose their existence over the whole of the finite complex plane, except in parts of the work in which their existence is assumed only on a line through the origin, taken without loss of generality as the real axis. Much of the development will be seen to be applicable on either hypothesis.

From the exchange of $x$ and $y$ in $(A)$ it appears that

$$
\phi(x-y)=-\phi(y-x), \quad \psi(x-y)=\psi(y-x) ;
$$

in other words, $\phi(x)$ is an odd and $\psi(x)$ an even function. Since $\phi(0)=0$, we obtain by putting $y=0$ in (2) either $\psi^{2}(0)=1$ or $\psi(x) \equiv 0$. If $\psi(x) \equiv 0$, it follows from (2) that $\phi(x) \equiv 0$, and this is also true if

$$
\psi(x) \equiv \pm 1
$$

We put to one side these trivial solutions, which are, indeed, the only ones in which both functions are constants.

It is immaterial whether we take $\psi(0)=1$ or $\psi(0)=-1$ since the four pairs of functions $\pm \phi(x), \pm \psi(x)$ simultaneously satisfy $(A)$. We take henceforth $\psi(0)=1$. Other pairs of functions satisfying $(A)$ are $\pm i \phi(x), \pm \psi(x)$. These eight solutions will be called equivalent.

The key to the properties of the two functions is to be found in the distribution of their zeros. We will first show that $\phi(x)$ and $\psi(x)$ can not vanish simultaneously for any value of $x$. Suppose, if possible, that they both vanish for $x=\xi$. Then on placing $y=x+\xi$ equations $(A)$ become

$$
\begin{aligned}
& 0=\phi^{2}(x) \psi^{2}(x+\xi)-\phi^{2}(x+\xi) \psi^{2}(x), \\
& 0=\psi^{2}(x) \psi^{2}(x+\xi)-\phi^{2}(x+\xi) \phi^{2}(x) .
\end{aligned}
$$

Since $\psi(x)$ is by hypothesis continuous and takes the value 1 at the origin, we can choose a positive $\delta$ so small that $\psi(x)$ will not vanish in the circle (interval) $|x| \leqq 2 \delta$. If either $\phi(x+\xi)$ or $\psi(x+\xi)$ vanishes for a value of $x$ in this circle (interval), equations (3) show that the other does also. When neither vanishes, their elimination from (3) gives $\phi^{4}(x)=\psi^{4}(x)$. Now for $y=x$ equation (2) yields the fundamental relation

$$
\psi(2 x)=\psi^{4}(x)-\phi^{4}(x),
$$

from which we get $\psi(2 x)=0$. This, however, is impossible for $|x| \leqq \delta$. It follows therefore that if $\phi(x)$ and $\psi(x)$ both vanish at any point $\xi$, they must vanish over a circle (interval) of radius $\delta$ having $\xi$ as its center. Follow now our functions along a straight line from $\xi$ to the origin. The interval in which both functions simultaneously vanish can not terminate at a point $\xi^{\prime}$ 
between $\xi$ and 0 because then both functions in consequence of their continuity would vanish at $\xi^{\prime}$ and hence in some circle (interval) having $\xi^{\prime}$ as its center. On the other hand, the interval can not extend to the origin because $\psi(0)=1$. From the resulting contradiction we conclude that the two functions can nowhere vanish at the same point.

We will next establish that when $\phi(x), \psi(x)$ exist over the complex plane, one or the other must have a zero not at the origin. For suppose the contrary and consider the quotient $R(x)=\phi(x) / \psi(x)$ which satisfies the functional equation

$$
R(x+y) R(x-y)=\frac{R^{2}(x)-R^{2}(y)}{1-R^{2}(x) R^{2}(y)} .
$$

By the hypothesis just made $R(x)$ is both finite and continuous, vanishing only for $x=0$. The right-hand member of $(B)$ is indeterminate only if $R^{2}(x)=R^{2}(y)= \pm 1$. It is, however, impossible for $R^{2}(x)$ to take the values \pm 1 , for we would then have $\phi^{4}(x)=\psi^{4}(x)$ and hence also $\psi(2 x)=0$, contrary to the hypothesis. Furthermore, $R(x)$ can not take the same value at two different points $x, y$ because then by $(B)$ we would have $R(x+y)=0$, i. e., $y=-x$, which is impossible since then $R(y)=R(-x)=-R(x)$. Consequently $R(x)$ must build the complex $x$-plane by a continuous 1-1 correspondence upon a portion of the $R$-plane.

In this correspondence a simply connected region including the origin of the $x$-plane is built upon a simply connected region including the origin of the $R$-plane. To the continuous set of circles of increasing radius about the $x$-origin corresponds a succession of closed contours about the origin of the $R$-plane which fill an open region $T$ which is the image of the finite $x$-plane. If $T$ does not extend infinitely far outwards from the origin $O$ in every direction save in the directions of the points $x= \pm 1, \pm i$, there must be in some other direction a first frontier point $\bar{R}$. On $O \bar{R}$ we can select points $R(x)$, $R(y)$ so close to $\bar{R}$ that the corresponding points $x, y$ lie on circles of an arbitrarily large radius about the origin, and we can at the same time take $|y|$ so large relatively to $|x|$ that $|x+y|$ and $|x-y|$ will both be as large as we please. On the other hand, when $R(x)$ and $R(y)$ are sufficiently near to $\bar{R}$ and therefore to each other, it follows from $(B)$ that either $|R(x+y)|$ or $|R(x-y)|$, and hence either $|x+y|$ or $|x-y|$, must be extremely small. Since this gives a contradiction, the frontier point $\bar{R}$ can not exist. Then $T$ must stretch out to infinity in every direction from the origin except toward $\pm 1, \pm i$. Consequently within $T$ we can find points for which $R(x) R(y)= \pm 1$, whence it follows by $(B)$ that either $R(x+y)$ or $R(x-y)$ must be infinite. But this is impossible if always $\psi(x) \neq 0$. Thus finally we must reject the hypothesis that neither $\phi(x)$ nor $\psi(x)$ vanishes excepting the former at $x=0$. 
Suppose now $\alpha \neq 0$ to be a root of $\phi(x)$. For $y=x+\alpha$ equation (1) gives

$$
\frac{\phi^{2}(x)}{\psi^{2}(x)}=\frac{\phi^{2}(x+\alpha)}{\psi^{2}(x+\alpha)}
$$

Consequently $\alpha$ is a period of $\phi^{2}(x) / \psi^{2}(x)$, and $\phi(x)$ which vanishes for $x=0$ has the periodic set of zeros $\pm n \alpha$. If $\phi(x)$ is analytic, its complete system of zeros is simply or doubly periodic unless $\phi(x)$ vanishes over the entire plane. When $\phi(x)$ is merely supposed to be continuous, the same conclusion follows by a well-known argument* provided there is a finite lower limit for the absolute values of all zeros other than $\alpha=0$. If there is no such lower limit, then either the zeros for sufficiently small value of their modulus will all have the same argument $(\bmod . \pi)$ or we can form doubly periodic systems of zeros, for which the sides of the period parallelogram are smaller than any arbitrarily assigned magnitude. In the latter case the zeros will be everywhere dense and $\phi(x)$ will vanish over the entire plane. In the former case there is a line through the origin on which there exists arbitrarily small periods for the zeros and on which accordingly $\phi(x)$ everywhere vanishes. If, furthermore, $\phi(x)$ has a zero without this line, then from the periodicity of $\phi^{2}(x) / \psi^{2}(x)$ it follows that there is a set of equidistant parallel lines on which $\phi(x)$ everywhere vanishes.

Suppose next that $\psi(x)$ has a root $\beta$. For $y=x+\beta$ equation (2) becomes

$$
\frac{\phi^{2}(x)}{\psi^{2}(x)}=\frac{\psi^{2}(x+\beta)}{\phi^{2}(x+\beta)} .
$$

Consequently $\phi^{2}(x) / \psi^{2}(x)$ has the period $2 \beta$, and $2 \beta$ must be a zero of $\phi(x)$. Since also either $\phi(x)$ or $\psi(x)$ possesses a root not at the origin, we conclude that $\phi(x)$ has always one such zero and therefore a periodic system of zeros. Furthermore, from (6) it is evident that the zeros of either function are obtained from those of the other by a displacement $\beta$. Thus if $\psi(x)$ has a single zero, the system of zeros of $\phi(x)$ and $\psi(x)$ must be of the same character. Later it will be shown $(\S 5)$ that if the zeros of $\phi(x)$ are isolated and either simply or doubly periodic, $\psi(x)$ will possess zeros also.

According to the nature of the root system of $\phi(x)$ the following four cases may now be distinguished, and only these:

(1) $\phi(x) \equiv 0$.

(2) $\phi(x)$ vanishes everywhere upon some straight line through the origin or over a set of equidistant parallel straight lines, one of which passes through $x=0$.

(3) $\phi(x)$ and $\psi(x)$ have each a simply periodic set of zeros, those of the

\footnotetext{
* Cf. Osgood's Funktionentheorie, 2d ed., pp. 459-460.
} 
latter function being situated half way between consecutive zeros of the former, inasmuch as $2 \beta$ is a root of $\phi(x)$.

(4) Both functions have a doubly periodic set of zeros. Three positions are possible for the zeros of $\psi(x)$ relatively to those of $\phi(x)$; they may be either the centers of the period-parallelograms for the zeros of $\phi(x)$, or the middle points of one system of parallel sides, or the middle points of the other.

\section{A special Functional EQUation}

We shall consider first the case in which $\phi(x)$ vanishes over the entire complex plane. Equation (2) then reduces to the functional equation

$$
\psi(x+y) \psi(x-y)=\psi^{2}(x) \psi^{2}(y) .
$$

More generally, this equation holds for any line through the origin on which $\phi(x)$ vanishes identically.

Consider now any such line and assume for $\psi(x)$ an arbitrary value at some initial point $x=x^{\prime}$ of the line. Unless $\psi(x)$ vanishes over the entire line, we can take a point for which the initial value is not zero. Seek now to determine the form of $\psi(x)$ on the line.

For $y=x$ we have

$$
\psi(2 x)=\psi^{4}(x)
$$

since we may take $\psi(0)=1$, precisely as in $\S 1$.

The formula

$$
\psi(n x)=[\psi(x)]^{n^{2}}
$$

can now be established for positive integral values of $n$ by mathematical induction. For assume it to hold up to a particular value of $n$. When $y=n x$, equation $(C)$ gives

$$
\psi[(n+1) x]=\frac{\psi^{2}(n x) \psi^{2}(x)}{\psi[(n-1) x]}=\frac{[\psi(x)]^{2 n^{2}+2}}{[\psi(x)]^{(n-1)^{2}}}=[\psi(x)]^{(n+1)^{2}} .
$$

Hence (8) continues to hold when $n$ is replaced by $n+1$.

From (8) we know $\psi(x)$ over the point set $\left\{n x^{\prime}\right\},(n=2,3, \cdots)$. Let $x$ in (7) be now replaced by $x^{\prime} / 2$. We get

$$
\psi\left(\frac{x^{\prime}}{2}\right)=\left[\psi\left(x^{\prime}\right)\right]^{1 / 4},
$$

whence by repeatedly halving the argument we obtain

$$
\psi\left(\frac{x^{\prime}}{2^{m}}\right)=\left[\psi\left(x^{\prime}\right)\right]^{1 / 2^{2 m}} . \quad(m=1,2, \cdots) .
$$

In the successive applications of (9) to halve the argument any succession of 
fourth roots can be chosen at the start, but ultimately the choice is restricted by the requirement that as $x^{\prime} / 2^{m}$ approaches the origin, $\psi\left(x^{\prime} / 2^{m}\right)$ shall approach the limit $\psi(0) \equiv 1$. Hence when $x^{\prime}$ in (9) is replaced by $x^{\prime} / 2^{m}$, we must select in the right-hand member for sufficiently large values of $m$ that fourth root whose argument is most nearly equal to 0 . Then from and after some fixed value $m=m^{\prime}$ the arguments of the successive fourth roots will be each a fourth of the preceding argument. Hence if we substitute $x^{\prime} / 2^{m \prime}$ in place of $x^{\prime}$ for our initial point but still denote it by $x^{\prime}$, all ambiguity in the determination of $\psi\left(x^{\prime} / 2^{m}\right)$ will be removed. Then by (8) and (10) we have

$$
\psi\left(\frac{n x^{i}}{2^{m}}\right)=\left[\psi\left(x^{\prime}\right)\right]^{\left(n / 2^{m}\right)^{2}} .
$$

Thus our function is uniquely determined over the dense set $\left\{n x^{\prime} / 2^{m}\right\}$ on one half of the line, and a like determination for the other half results from the relation $\psi(-x)=\psi(x)$. Being continuous, the function is thereby completely determined, provided only the values obtained over the dense pointset are consistent with the requirement of continuity.

To establish this consistency choose a value of $c$ such that $e^{c x^{2}}$ equals the assigned initial value and has the same argument. By substitution of $e^{c x^{2}}$ for $\psi(x)$ in $(C)$ it will be found that the equation is satisfied. The function $e^{c x^{2}}$ also fulfills the requirement that when $x$ is replaced by $x / 2$, its argument is divided by 4 . We conclude therefore, that unless $\psi(x)$ vanishes along the entire line, it must have the form $e^{c x^{2}}$.

Proceed next to the determination of the solution over the complex plane. Should $\psi(x)$ vanish along one line through the origin and be of form $e^{c x^{2}}$ on another such line, there would be two conflicting values at $x=0$. Hence if $\psi(x)$ vanishes at a single point, it must vanish over the entire plane. Assume now for $\psi(x)$ arbitrary values different from zero at three initial points $x^{\prime}, y^{\prime}, x^{\prime}-y^{\prime}$ which do not lie on a common line through the origin. Its value at $x^{\prime}+y^{\prime}$ is then determined by $(C)$. By putting in this equation $y=y^{\prime}, x=x^{\prime}+n y^{\prime}(n=1,2, \cdots)$ we determine $\psi(x)$ successively at the points $x^{\prime}+(n+1) y^{\prime}$, and similarly at $y^{\prime}+(n+1) x^{\prime}$. Equation (8) gives $\psi\left(n x^{\prime}\right)$ and $\psi\left(n y^{\prime}\right)$. Then placing $y=x^{\prime}, x=n x^{\prime}+m y^{\prime}(n=1$, $2, \cdots)$ in $(C)$ we get $\psi(x)$ at all remaining points of form $n x^{\prime}+m y^{\prime}$. Its values at $n x^{\prime}-m y^{\prime}$ may next be found by replacing $x, y$ in $(C)$ by $n x^{\prime}, m y^{\prime}$. Lastly, $\psi\left(-n x^{\prime} \pm m y^{\prime}\right)=\psi\left(n x^{\prime} \mp m y^{\prime}\right)$. Thus $\psi(x)$ is uniquely determined over the periodic network $\pm m x^{\prime} \pm n y^{\prime}$ in terms of its values at the three initial points.

As before, equation (9) may be used to halve successively the initial arguments $x^{\prime}, y^{\prime}$ and $x^{\prime}-y^{\prime}$, the choice of the fourth root in the right-hand 
member being ultimately unique. If now for a sufficiently large value $m^{\prime}$ we substitute $x^{\prime} / 2^{m \prime}, y^{\prime} / 2^{m \prime},\left(x^{\prime}-y^{\prime}\right) / 2^{m \prime}$ with the corresponding values of $\psi$ in place of the initial points and values but retain the old notation $x^{\prime}, y^{\prime}$, $x^{\prime}-y^{\prime}$ for the new initial points, all ambiguity disappears. Then $\psi(x)$ will be known uniquely over the dense set of points

$$
\frac{n x^{\prime}}{2^{m}}+\frac{n^{\prime} y^{\prime}}{2^{m \prime}} \quad\left(m, n, m^{\prime}, n^{\prime}=0, \pm 1, \pm 2, \cdots\right)
$$

and therefore over the entire plane, provided that the values over this pointset are consistent with the requirement of continuity for $\psi(x)$.

To settle this question we will set up an actual solution of $(C)$ which will take arbitrarily assumed values at the initial points. Put

$$
x=u+i v,
$$

and take for $\psi(x) \equiv \psi(u+i v)$ the function

$$
e^{c_{1} u^{2}+c_{2} u v+c_{8} v^{2}}
$$

in which the $c_{i}$ denote arbitrary constants, real or complex. By direct substitution in $(C)$ it will be found that the functional equation is satisfied. Let $e^{k_{1}}, e^{k_{2}}, e^{k_{3}}$ denote the values assigned arbitrarily at the initial points

$$
x^{\prime} \equiv u_{1}+i v_{1}, \quad y^{\prime} \equiv u_{2}+i v_{2}, \quad \text { and } \quad x^{\prime}-y^{\prime} .
$$

The expression (11) will take these values if

$$
\begin{aligned}
& k_{1}=c_{1} u_{1}^{2}+c_{2} u_{1} v_{1}+c_{3} v_{1}^{2}, \\
& k_{2}=c_{1} u_{2}^{2}+c_{2} u_{2} v_{2}+c_{3} v_{2}^{2}, \\
& k_{3}=c_{1}\left(u_{1}-u_{2}\right)^{2}+c_{2}\left(u_{1}-u_{2}\right)\left(v_{1}-v_{2}\right)+c_{3}\left(v_{1}-v_{2}\right)^{2} .
\end{aligned}
$$

The determinant of this system of equations for $c_{1}, c_{2}, c_{3}$ is

$$
\left|\begin{array}{ccc}
u_{1}^{2} & u_{1} v_{1} & v_{1}^{2} \\
u_{2}^{2} & u_{2} v_{2} & v_{2}^{2} \\
\left(u_{1}-u_{2}\right)^{2} & \left(u_{1}-u_{2}\right)\left(v_{1}-v_{2}\right) & \left(v_{1}-v_{2}\right)^{2}
\end{array}\right| \equiv\left(u_{1} v_{2}-v_{1} u_{2}\right)^{3},
$$

which vanishes only if $u_{1}+i v_{1}$ and $u_{2}+i v_{2}$ lie on a line which passes through the origin. As this is contrary to hypothesis, the expression (11) can be made to take the required values.

Besides our solution $\psi(x)$ there is an equivalent solution, $-\psi(x)$. Thus the expression (11) and its negative gives the general expression for all continuous solutions of $(C)$ except the trivial solution $\psi(x)=0$. If, in particular, the solution is analytic, it has the form $\psi(x)= \pm e^{c x^{2}}$. 
The functional equation $(C)$ comes a second time into the investigation when a common factor $s(x)$ is sought by which $\phi(x), \psi(x)$ can be modified without ceasing to be a solution of $(A)$. For let them be replaced in either equation of $(A)$ by $s(x) \phi(x), s(x) \psi(x)$ and divide the resulting equation by the same equation before the substitution. Thereby we obtain $(C)$ with $s(x)$ in place of $\psi(x)$. The division becomes impossible for both pairs of equations only if

or

$$
\phi(x+y)=0, \quad \psi(x-y)=0,
$$

$$
\phi(x-y)=0, \quad \psi(x+y)=0 .
$$

Hence at most $s(x)$ can fail to satisfy $(C)$ only for exceptional pairs of values $(x, y)$ which satisfy one of these pairs of equations. Now because of the periodicity of their roots neither $\phi(x)$ nor $\psi(x)$ vanishes over any region of the $x$-plane unless it vanishes everywhere. Hence in the vicinity of any exceptional pair $(x, y)$ there are values for which $(C)$ is satisfied by $s(x)$, whence it follows by continuity that the equation is also satisfied for these exceptional values. We conclude therefore that $\phi(x), \psi(x)$ can be multiplied by a continuous common factor without ceasing to be a solution of $(A)$ when and only when the factor is of form (11).

\section{Solutions WiTH LINES OF ZEROS}

The case in which $\phi(x)$ possesses one or more lines on which it vanishes identically can be quickly disposed of. Let $\alpha$ denote a zero lying upon the line of zeros through the origin. From (5) it follows that the ratio

$$
R^{2}(x+\alpha) \equiv \phi^{2}(x+\alpha) / \psi^{2}(x+\alpha)
$$

for fixed $x$ must remain constant when $\alpha$ continuously describes the line of zeros. Consequently $R^{2}(x)$ remains constant when $x$ describes any straight line parallel to the line of zeros, and this must likewise be true of $R(x)$ which can not change sign abruptly

For convenience suppose the line of zeros not to coincide with the real axis. If necessary, this can be avoided by a rotation of the plane around the origin which in no wise affects the form of $(A)$. Then any solution in which $\phi(x)$, $\psi(x)$ fulfill the above requirement of having a constant ratio on every line parallel to the line of zeros can evidently be constructed as follows. Start with its section $\phi(u), \psi(u)$ along the real axis and construct functions $\bar{\phi}(x), \bar{\psi}(x)$ which coincide with this section along the real axis and are constant on every line parallel to the line of zeros. Clearly $\bar{\phi}(x), \bar{\psi}(x)$ satisfy $(A)$. The solution can then be obtained by modifying these func- 
tions by a common factor, which can only be of form (11). The zeros of $\phi(x), \psi(x)$ on the real axis give rise to the lines of zeros.

The following possibilities occur:

(1) $\phi(x)$ possesses only a line of zeros through the origin.

(2) $\phi(x)$ possesses a set of equidistant lines of zeros, while $\psi(x)$ has no zeros.

(3) Both $\phi(x)$ and $\psi(x)$ possess a set of equidistant lines of zeros, the lines for either function being half way between two consecutive lines for the other function.

The real solutions given in $\S 10$ give rise to solutions of the $2 \mathrm{~d}$ and $3 \mathrm{~d}$ kinds, while (1) can be obtained from suitable unreal solutions.

Since every solution with lines of zeros can be constructed in the above very simple manner from a section of some solution along the axis or other line through the origin, the case is essentially trivial and is solved when the other cases are solved. Hence it need not be considered further.

\section{The NORMalization OF THE solutions}

It may be henceforth supposed that the zeros of $\phi(x)$ form a discrete system, either simply or doubly periodic. Let $\alpha$ be a primitive zero, that is, one so situated that no zero lies in the segment $(0, \alpha)$. Place

$$
\phi(x+\alpha)=t(x) \phi(x),
$$

where $t(x)$ is a function to be determined. From (5) and (13a) we have

$$
\psi(x+\alpha)= \pm t(x) \psi(x) .
$$

A comparison of the last two equations shows that the sign in the right-hand member of the latter equation cannot vary with $x$ inasmuch as $\phi(x+\alpha) / \phi(x)$ and $\psi(x+\alpha) / \psi(x)$ are continuous except at the zeros of their respective denominators which do not simultaneously vanish. Then $t(x)$ must be continuous over the entire $x$-plane.

To determine $t(x)$ replace $x$ by $x+\alpha$ in either equation of $(A)$ and divide either resulting equation by the same equation before the replacement. By application of (13) we obtain in either case

$$
t(x+y) t(x-y)=t^{2}(x) .
$$

The division fails only for values of the arguments which satisfy $\left(12^{\prime}\right)$ or $\left(12^{\prime \prime}\right)$, and the equation can be extended to these arguments also by a proper limiting passage, as in $\S 2$. Equation (14) gives

$$
t(2 x) t(0)=t^{2}(x),
$$


and hence on placing $f(x)=t(x) / t(0)$ it can be reduced to the well-known form

$$
f(x) f\left(x^{\prime}\right)=f\left(x+x^{\prime}\right) .
$$

Any solution of this equation which is continuous along the real axis and does not vanish identically was shown by Cauchy to have the form $e^{c x}$ along the $x$-axis, from which it follows that

$$
f(u+i v)=f(u) f(i v)=e^{c^{\prime} u+c^{\prime \prime} v} .
$$

Thus we have as the general solution of (14)

$$
t(x) \equiv t(u+i v)=c e^{c^{\prime} u+c^{\prime \prime} v},
$$

in which $c, c^{\prime}, c^{\prime \prime}$ denote arbitrary constants.

Equation (13a) may now be written

$$
\phi(u+i v+\alpha)=c e^{c^{\prime} u+c^{\prime \prime} v} \phi(u+i v)
$$

with a corresponding equation $(16 b)$ for $\psi(u+i v)$. The three constants here are not independent but if $\alpha=\alpha^{\prime}+i \alpha^{\prime \prime}$, are bound by the relation

$$
c=-e^{\frac{1}{(1}\left(c^{\prime} \alpha^{\prime}+c^{\prime \prime} \alpha^{\prime \prime}\right)}
$$

obtained by putting $u+i v=-\alpha / 2$.

Our equations (16) may now be normalized by using the property that $\phi(x), \psi(x)$ remain a solution of $(A)$ when modified by an arbitrary factor of form (11). If $\phi(x)$ is replaced by $e^{c_{1} u^{2}+c_{2} u v} \phi(x)$, equation (16a) thereby becomes

$$
e^{c_{1}\left(2 a^{\prime} u+\alpha^{\prime 2}\right)+c_{2}\left(\alpha^{\prime} v+\alpha^{\prime \prime} u+\alpha^{\prime} a^{\prime \prime}\right)} \phi\left(u+i v+\alpha^{\prime}+i \alpha^{\prime \prime}\right)=c e^{c^{\prime} u+c^{\prime \prime} v} \phi(u+i v) .
$$

Unless* $\alpha^{\prime}=0$, the arbitraries $c_{1}, c_{2}$ can be so chosen that the exponential term on the right is canceled out. Then in consequence of (17) our equation reduces to

$$
\phi(u+i v+\alpha)=-\phi(u+i v) .
$$

Thus by normalization (16) may be thrown into one of two forms, either

$$
\phi(x+\alpha)=-\phi(x), \quad \psi(x+\alpha)=-\psi(x),
$$

$$
\phi(x+\alpha)=-\phi(x), \quad \psi(x+\alpha)=\psi(x) .
$$

In similar manner, if $\beta$ is a primitive root of $\psi(x)$, we may place in accord with (6)

\footnotetext{
* In which case we can obtain the same result by using $e^{c_{2} u v+c_{3} v^{2}} \phi(x)$.
} 


$$
\begin{aligned}
& \phi(x+\beta)=t(x) \psi(x), \\
& \psi(x+\beta)= \pm t(x) \phi(x),
\end{aligned}
$$

in which $t(x)$ denotes again a continuous function to be determined. If $x$ is replaced by $x+\beta$ in $(A)$ and either equation after the replacal is divided by the other equation before the replacal, equation (14) is again obtained for $t(x)$. Hence

$$
\begin{aligned}
& \phi(x+\beta)=b e^{b_{1} u+b_{2} v} \psi(x), \\
& \psi(x+\beta)= \pm b e^{b_{1} u+b_{2} v} \phi(x)
\end{aligned}
$$

$$
(x=u+i v) .
$$

Here, as in $(13 b)$, the sign before $b$ in the second right-hand member is independent of $x$, and for like reason. From (4) we have $\phi^{4}(\beta / 2)=\psi^{4}(\beta / 2)$, and hence if $\beta=\beta^{\prime}+i \beta^{\prime \prime}$, we obtain by putting $x=-\beta / 2$

$$
b^{4}=e^{2\left(\beta^{\prime} b_{1}+\beta^{\prime \prime} b_{2}\right)} \text {. }
$$

By normalizing (20) in the same manner as (16), though not simultaneously unless $\alpha=2 \beta$, we may remove the exponential terms. Thereafter we have

$$
\begin{aligned}
& \phi(x+\beta)=l \psi(x), \\
& \psi(x+\beta)= \pm l \phi(x)
\end{aligned}
$$

These equations are consistent for $x=-\beta / 2$ only if $l^{2}=\mp 1$. By making a proper choice among the eight equivalent solutions they may be given the definite form:

$$
\begin{aligned}
& \phi(x+\beta)=\psi(x), \\
& \psi(x+\beta)=-\phi(x) .
\end{aligned}
$$

The last pair of equations as well as the one immediately preceding gives

$$
\phi(x+2 \beta)=-\phi(x), \quad \psi(x+2 \beta)=-\psi(x) .
$$

From this it follows that equations (18) and not (19) must be chosen when $\alpha / 2$ is a root of $\psi(x)$. Conversely, when (18) holds, $\alpha / 2$ must be a root of $\psi(x)$, as is apparent on placing $x=-\alpha / 2$ in (18). Thus the choice between (18) and (19) is settled by the presence or absence of a root of $\psi(x)$ at $\alpha / 2$.

By means of our normalized formulas we may now derive a formula for $\phi(2 x)$. Suppose first that $\alpha / 2$ is not a root of $\psi(x)$ so that (19) comes into play. Upon replacing $x, y$, in (1) by $x+\alpha / 4, x-\alpha / 4$ we get

$$
\begin{aligned}
\phi\left(\frac{\alpha}{2}\right) \phi(2 x)=\phi^{2}\left(x-\frac{3 \alpha}{4}\right) \psi^{2}\left(x+\frac{3 \alpha}{4}\right) & \\
& -\phi^{2}\left(x+\frac{3 \alpha}{4}\right) \psi^{2}\left(x-\frac{3 \alpha}{, 4}\right) .
\end{aligned}
$$


Also from $(A)$ we have

$$
\begin{aligned}
& \pm \phi\left(x \pm \frac{3 \alpha}{4}\right) \phi\left(\frac{\alpha}{4}\right)=\phi^{2}\left(\frac{x}{2} \pm \frac{\alpha}{2}\right) \psi^{2}\left(\frac{x}{2} \pm \frac{\alpha}{4}\right)-\psi^{2}\left(\frac{x}{2} \pm \frac{\alpha}{2}\right) \dot{\phi}^{2}\left(\frac{x}{2} \pm \frac{\alpha}{4}\right) \\
& \psi\left(x \pm \frac{3 \alpha}{4}\right) \psi\left(\frac{\alpha}{4}\right)=\psi^{2}\left(\frac{x}{2} \pm \frac{\alpha}{2}\right) \psi^{2}\left(\frac{x}{2} \pm \frac{\alpha}{4}\right)-\phi^{2}\left(\frac{x}{2} \pm \frac{\alpha}{2}\right) \phi^{2}\left(\frac{x}{2} \pm \frac{\alpha}{4}\right)
\end{aligned}
$$

By the use of these we get for one factor of the right-hand member of (23)

$$
\begin{gathered}
\phi\left(x+\frac{3 \alpha}{4}\right) \psi\left(x-\frac{3 \alpha}{4}\right)+\phi\left(x-\frac{3 \alpha}{4}\right) \psi\left(x+\frac{3 \alpha}{4}\right) \\
=\frac{1}{\phi\left(\frac{\alpha}{4}\right) \psi\left(\frac{\alpha}{4}\right)}\left[\psi^{4}\left(\frac{x}{2}+\frac{\alpha}{2}\right)-\phi^{4}\left(\frac{x}{2}+\frac{\alpha}{2}\right)^{1}\right] \\
\cdot\left[\phi^{2}\left(\frac{x}{2}-\frac{\alpha}{4}\right) \psi^{2}\left(\frac{x}{2}+\frac{\alpha}{4}\right)-\phi^{2}\left(\frac{x}{2}+\frac{\alpha}{4}\right) \psi^{2}\left(\frac{x}{2}-\frac{\alpha}{4}\right)\right] \\
=\frac{-\psi(x) \phi(x) \phi\left(\frac{\alpha}{2}\right)}{\phi\left(\frac{\alpha}{4}\right) \psi\left(\frac{\alpha}{4}\right)} .
\end{gathered}
$$

The other factor of the right-hand member by virtue of (19) is identical with the first factor, after $x$ is replaced by $x+\alpha / 2$ in the latter factor. Hence we have

$$
\phi(2 x)=\frac{\phi\left(\frac{\alpha}{2}\right)}{\phi^{2}\left(\frac{\alpha}{4}\right) \psi^{2}\left(\frac{\alpha}{4}\right)} \phi(x) \psi(x) \phi\left(x+\frac{\alpha}{2}\right) \psi\left(x+\frac{\alpha}{2}\right) .
$$

But for $x=\alpha / 2$ equation (24) gives

$$
2 \phi\left(\frac{\alpha}{4}\right) \psi\left(\frac{\alpha}{4}\right)=\frac{\phi^{2}\left(\frac{\alpha}{2}\right) \psi\left(\frac{\alpha}{2}\right)}{\phi\left(\frac{\alpha}{4}\right) \psi\left(\frac{\alpha}{4}\right)}
$$

so that the preceding equation takes the final form

$$
\phi\left(\frac{\alpha}{2}\right) \psi\left(\frac{\alpha}{2}\right) \phi(2 x)=2 \phi(x) \psi(x) \phi\left(x+\frac{\alpha}{2}\right) \psi\left(x+\frac{\alpha}{2}\right)
$$

The reader will observe the identity in structure with the familiar formula for the Jacobian function $\vartheta_{1}(2 x)$. It holds when $\alpha / 2$ is not a root of $\psi(x)$ and the normalization of the functions is made with reference to the root $\alpha$. 
Suppose, on the other hand, that $\beta \equiv \alpha / 2$ is a root of $\psi(x)$, and select from the equivalent normalized solutions one for which (22) is valid. Then by (22) we have

$$
\phi\left(\frac{\alpha}{2}\right)=\psi(0)=1, \quad \phi\left(\frac{\alpha}{4}\right)=\psi\left(\frac{\alpha}{4}\right),
$$

and by (22) and (4)

$$
\phi(2 x)=-\psi\left(2 x+\frac{\alpha}{2}\right)=-\psi^{4}\left(x+\frac{\alpha}{4}\right)+\phi^{4}\left(x+\frac{\alpha}{4}\right) .
$$

By means of (26) and the relation

$\phi(x) \psi(x)=\phi(x) \phi\left(x+\frac{\alpha}{2}\right)=\phi^{2}\left(x+\frac{\alpha}{4}\right) \psi^{2}\left(\frac{\alpha}{4}\right)-\phi^{2}\left(\frac{\alpha}{4}\right) \psi^{2}\left(x+\frac{\alpha}{4}\right)$

the last equation may be thrown into the form

$$
\phi(2 x)=\frac{\phi(x) \psi(x)}{\phi^{2}\left(\frac{\alpha}{4}\right)}\left[\phi^{2}\left(x+\frac{\alpha}{4}\right)+\psi^{2}\left(x+\frac{\alpha}{4}\right)\right] .
$$

When $\phi^{2}+\psi^{2} \equiv 1$, this formula is identical in structure with that for $\sin 2 x$.

\section{ON THE EXISTENCE OF ZEROS OF $\psi(x)$}

The existence of a zero of $\psi(x)$, and hence of a simply or doubly periodic system of zeros like that of $\phi(x)$, can now be established.

Suppose first the zeros of $\phi(x)$ form a doubly periodic network, and let $\alpha, \alpha^{\prime}$ be a primitive pair of zeros. If $\alpha / 2$ is not a root of $\psi(x)$, we may make use of the normalized equation (25). For $x=\alpha^{\prime} / 2$ this becomes

$$
0=\phi\left(\frac{\alpha^{\prime}}{2}\right) \psi\left(\frac{\alpha^{\prime}}{2}\right) \phi\left(\frac{\alpha+\alpha^{\prime}}{2}\right) \psi\left(\frac{\alpha+\alpha^{\prime}}{2}\right) .
$$

Hence $\psi(x)$ must vanish at $\alpha^{\prime} / 2$ or $\left(\alpha+\alpha^{\prime}\right) / 2$ if it does not vanish at $\alpha / 2$.

When the zeros of $\phi(x)$ form only a simply periodic system, the existence of a zero of $\psi(x)$ can be readily established in the following manner similar to that in which a zero was proved to exist for either $\phi(x)$ or $\psi(x)$ in $\S 1$. Suppose, if possible, $\psi(x)$ to have no root. Then by (19) we have

$$
R(x+\alpha)=-R(x) .
$$

Through $\alpha / 2$ and $-\alpha / 2$ in the $x$-plane draw two parallel lines, and denote by $S$ a half of the resulting strip which lies to one side of the segment joining $\pm \alpha / 2$. Consider the representation of $S$ upon the $R$-plane effected through $R(x)$. As seen in $\S 1$, it is impossible for $R(x)$ to assume any of the 
values $\pm 1, \pm i$ if $\psi(x)$ has no zero, and $(B)$ has then always a sense. If $R(x)$ should take the same value at two different points $x, y$ of $S$, it would follow from $(B)$ that either $x+y$ or $x-y$ would be a root of $R(x)$ and hence would be equal to 0 or a multiple of $\alpha$, which necessarily is $\pm \alpha$ when $x, y$ lie in $S$. But clearly we cannot have $x-y= \pm \alpha$ because of (28), nor $x+y=0$ because $R(-x)=-R(x)$. Hence $R(x)$ will build the half-strip by a continuous 1-1 correspondence upon a simply connected region of the $R$-plane whose boundary passes through the origin. The other half-strip is built upon a like region which is the symmetrical image of the first with respect to the origin since $R(-x)=-R(x)$. A circle of radius $\alpha / 2$ about the origin of the $x$-plane by the same reasoning as was applied to $S$ will be built by continuous 1-1 correspondence upon a region inclosing the origin of the $R$-plane. Take in the latter region a circle $C^{\prime}$ of radius $\epsilon$ about the origin whose image will be a very small vicinity $C$ of $x=0$. Then $|R(x)|$ by (28) can be equal to or less than $\epsilon$ only when $x$ falls in $C$ or a congruent vicinity of one of the points $x= \pm n \alpha$.

Denote the $R$-image of $S$ by $S^{\prime}$. Any segment parallel to the base of $S$ and terminated by its parallel sides will be built upon some sort of a line of the $R$-plane whose terminal points lie without $C^{\prime}$ and which by (28) are symmetrically situated with respect to the origin. As the segment recedes to infinity, either its image will sweep over a region which extends to infinity or there will be frontier points $L$ of the region other than $\pm 1, \pm i$. In the vicinity of any such point $L$ we can select within $S^{\prime}$ a set of $R$-points with the limit $L$, for which the corresponding $x$-points recede to infinity. From these we can take two points $R(x), R(y)$ arbitrarily close to $L$ in such wise that $|y|$ will be as large as we please relatively to $|x|$, and in consequence both $|x+y|$ and $|x-y|$ will be extremely large. If, furthermore, $R(x)$ and $R(y)$ are sufficiently close to $L$ and therefore to each other, it follows by $(B)$ that either $|R(x+y)|$ or $|R(x-y)|$ may be made arbitrarily small. Accordingly, either $x+y$ or $x-y$ may be made to fall within $C$, or within a congruent vicinity of one of the points $\pm \alpha$. But obviously this is impossible if both $|x+y|$ and $|x-y|$ are very large. From this contradiction it results that $S^{\prime}$ must stretch out to infinity. Then it must contain points $R(x)$ such that

$$
1 /|R(x)|<\epsilon,
$$

and hence points $R(x), R(y)$ for which $R(x) R(y)=1$. It follows then by $(B)$ that either $R(x+y)$ or $R(x-y)$ must be infinite. But this is impossible unless $\psi(x+y)=0$ or $\psi(x-y)=0$. Consequently the supposition that $\psi(x)$ has no zero is untenable. Having one zero, it has a doubly or simply periodic set of zeros simultaneously with $\phi(x)$. 


\section{The ALlied solutions}

We seek next to find non-equivalent solutions whose first components $\phi(x)$ differ only by a constant factor.

Suppose $\phi(x), \psi(x)$ to be any solution normalized according to (18) or (19). Then it may be shown quickly that

$$
\phi_{2}(x) \equiv \frac{\phi(x)}{\phi\left(\frac{\alpha}{2}\right)}, \quad \psi_{2}(x) \equiv \frac{\phi\left(x+\frac{\alpha}{2}\right)}{\phi\left(\frac{\alpha}{2}\right)} \equiv \phi_{2}\left(x+\frac{\alpha}{2}\right)
$$

is also a solution. For let these functions be substituted in $(A)$ in place of $\phi(x), \psi(x)$. The equations thereby become

$$
\begin{aligned}
& \phi^{2}\left(\frac{\alpha}{2}\right) \phi(x+y) \phi(x-y)=\phi^{2}(x) \phi^{2}\left(y+\frac{\alpha}{2}\right)-\phi^{2}(y) \phi^{2}\left(x+\frac{\alpha}{2}\right) \\
& \phi^{2}\left(\frac{\alpha}{2}\right) \phi\left(x+y+\frac{\alpha}{2}\right) \phi\left(x-y+\frac{\alpha}{2}\right) \\
&=\phi^{2}\left(x+\frac{\alpha}{2}\right) \phi^{2}\left(y+\frac{\alpha}{2}\right)-\phi^{2}(x) \phi^{2}(y) .
\end{aligned}
$$

The first of these passes into the second when $x$ is replaced by $x+\alpha / 2$. It suffices then to verify the truth of the former. Now

$$
\begin{aligned}
\phi^{2}\left(x+\frac{\alpha}{2}\right)=-\phi\left(x+\frac{\alpha}{2}\right) \phi & \left(x-\frac{\alpha}{2}\right) \\
& =-\phi^{2}(x) \psi^{2}\left(\frac{\alpha}{2}\right)+\phi^{2}\left(\frac{\alpha}{2}\right) \psi^{2}(x) .
\end{aligned}
$$

The introduction of this expression and of the like expression for $\phi^{2}(y+\alpha / 2)$ into (30) converts it into (1). Accordingly (29) furnishes a solution of $(A)$.

It may next be shown that if $\alpha / 2$ is not a zero of $\psi(x)$, then

$$
\frac{\phi(x)}{\sqrt{i} \psi\left(\frac{\alpha}{2}\right)}, \frac{\psi\left(x+\frac{\alpha}{2}\right)}{\psi\left(\frac{\alpha}{2}\right)}
$$

also constitute a solution of $(A)$. For by virtue of (19) we have

$$
\psi^{2}\left(x+\frac{\alpha}{2}\right)=\psi\left(x+\frac{\alpha}{2}\right) \psi\left(x-\frac{\alpha}{2}\right)=\psi^{2}(x) \psi^{2}\left(\frac{\alpha}{2}\right)-\phi^{2}(x) \phi^{2}\left(\frac{\alpha}{2}\right)
$$

If now the functions (33) are substituted in $(A)$ in place of $\phi(x), \psi(x)$, two 
equations are obtained, of which the first becomes identical with (1) by the aid of (34). The second is

$$
\begin{aligned}
\psi^{2}\left(\frac{\alpha}{2}\right) \psi\left(x+y+\frac{\alpha}{2}\right) \psi(x-y & \left.+\frac{\alpha}{2}\right) \\
& =\psi^{2}\left(x+\frac{\alpha}{2}\right) \psi^{2}\left(y+\frac{\alpha}{2}\right)+\phi^{2}(x) \phi^{2}(y) .
\end{aligned}
$$

If $x$ is here replaced by $x+\alpha / 2$, the equation reduces at once to (2) through the use of (32), (34), and (19). Hence (33) gives a solution, as stated.

Seek next every possible solution

$$
\phi_{i}(x) \equiv c_{i} \phi(x), \quad \psi_{i}(x),
$$

having the same first component save for a constant factor $c_{i}$. It should be carefully noted that this entire group of solutions is simultaneously normalized, inasmuch as a normalization of the $\phi$-component carries with it a normalization of the corresponding $\psi$. But equation (18) may hold for one solution and (19) for another, and a corresponding remark applies to (21).

By substitution of (35) in (1) and then by combination of the resulting equation with (1) we get

$$
\frac{\phi^{2}(x)}{\psi^{2}(x)-\psi_{i}^{2}(x)}=\frac{\phi^{2}(y)}{\psi^{2}(y)-\psi_{i}^{2}(y)} .
$$

As the arguments of the two members are independent, each member is a constant. Hence

$$
\psi_{i}^{2}(x)=\psi^{2}(x)+k_{i}^{2} \phi^{2}(x),
$$

in which $k_{i}^{2}$ is a constant to be subsequently determined. Take now (29) as the primary solution, for which we have

$$
\phi_{2}\left(\frac{\alpha}{2}\right)=1, \quad \psi_{2}\left(\frac{\alpha}{2}\right)=0, \quad \phi_{2}\left(\frac{\alpha}{4}\right)=\psi_{2}\left(\frac{\alpha}{4}\right)
$$

Our preceding equation becomes

$$
\psi_{i}^{2}(x)=\psi_{2}^{2}(x)+k_{i}^{2} \phi_{2}^{2}(x) \equiv \phi_{2}^{2}\left(x+\frac{\alpha}{2}\right)+k_{i}^{2} \phi_{2}^{2}(x) .
$$

For $x=\alpha / 2$ this gives

$$
k_{i}^{2}=\psi_{i}^{2}\left(\frac{\alpha}{2}\right)
$$

If $\psi_{i}(x)$ vanishes at $\alpha / 2$, we have $k_{i}=0, c_{i}^{4}=1$ and obtain the primary solution or an equivalent solution. Henceforth it may be assumed that $\psi_{i}(x)$ does not vanish at $\alpha / 2$ so that we have $k_{i} \neq 0$ and also, by (19), $\psi_{i}(\alpha)=1$. Then on replacing $\psi$ by $\psi_{i}$ in (4) and putting $x=\alpha / 2$ we have 


$$
1=k_{i}^{4}-c_{i}^{4} .
$$

If the functions (35) with $\phi(x)$ taken equal to $\phi_{2}(x)$ are substituted in the two equations of $(A)$, the first is satisfied regardless of the values of $k_{i}$ and $c_{i}$ in consequence of (37). If the second is squared and modified with the aid of (37) and (39), it becomes

$$
\begin{array}{r}
{\left[\phi_{2}^{2}\left(x+y+\frac{\alpha}{2}\right)+k_{i}^{2} \phi_{2}^{2}(x+y)\right] \cdot\left[\phi_{2}^{2}\left(x-y+\frac{\alpha}{2}\right)+k_{i}^{2} \phi_{2}^{2}(x-y)\right]} \\
=\left[\phi_{2}^{2}\left(x+\frac{\alpha}{2}\right) \phi_{2}^{2}\left(y+\frac{\alpha}{2}\right)+\phi_{2}^{2}(x) \phi_{2}^{2}(y)\right. \\
\left.+k_{i}^{2}\left\{\phi_{2}^{2}(x) \phi_{2}^{2}\left(y+\frac{\alpha}{2}\right)+\phi_{2}^{2}(y) \phi_{2}^{2}\left(x+\frac{\alpha}{2}\right)\right\}\right]^{2} .
\end{array}
$$

For $x=y=\alpha / 4$ this furnishes for $k_{i}^{2}$ the quadratic equation

$$
k_{i}^{4}+\left(2-\frac{1}{4 \phi_{2}^{8}\left(\frac{\alpha}{4}\right)}\right) k_{i}^{2}+1=0 .
$$

By a somewhat laborious calculation it will next be shown that either of the two values of $k_{i}^{2}$ will reduce (40) to an identity. Let $k_{i}^{4}$ be first eliminated by (41). Then the terms independent of $k_{i}^{2}$ will cancel by (31), (30), and (36). Equating the coefficients of $k_{i}^{2}$ we have left as the identity to be verified,

$$
\begin{array}{r}
\phi_{2}^{2}(x+y) \phi_{2}^{2}\left(x-y+\frac{\alpha}{2}\right)+\phi_{2}^{2}(x-y) \phi_{2}^{2}\left(x+y+\frac{\alpha}{2}\right) \\
=2\left[\phi_{2}^{2}\left(x+\frac{\alpha}{2}\right) \phi_{2}^{2}\left(y+\frac{\alpha}{2}\right)+\phi_{2}^{2}(x) \phi_{2}^{2}(y)\right] \\
\cdot\left[\phi_{2}^{2}(x) \phi_{2}^{2}\left(y+\frac{\alpha}{2}\right)+\phi_{2}^{2}(y) \phi_{2}^{2}\left(x+\frac{\alpha}{2}\right)\right] \\
-\left(2-\frac{1}{\left.4 \phi_{2}^{2}\left(\frac{\alpha}{4}\right)\right)}\right)\left\{\left[\phi_{2}^{2}(x) \phi_{2}^{2}\left(y+\frac{\alpha}{2}\right)+\phi_{2}^{2}(y) \phi_{2}^{2}\left(x+\frac{\alpha}{2}\right)\right]^{2}\right. \\
\left.-\phi_{2}^{2}(x+y) \phi_{2}^{2}(x-y)\right\}
\end{array}
$$

By (30) the last term reduces to

$$
-4\left[2-\frac{1}{4 \phi_{2}^{8}\left(\frac{\alpha}{4}\right)}\right] \phi_{2}^{2}(x) \phi_{2}^{2}(y) \phi_{2}^{2}\left(x+\frac{\alpha}{2}\right) \phi_{2}^{2}\left(y+\frac{\alpha}{2}\right) .
$$


To verify the identity we will eliminate the arguments of the left-hand member, introducing ultimately in their place the same arguments as appear on the right. To this end replace $x, y$ in (30) and (31) by $x+\alpha / 4, y-\alpha / 4$. Then squaring, adding, and using the relation

$$
\phi_{2}^{2}\left(x-\frac{\alpha}{4}\right)=\psi_{2}^{2}\left(x+\frac{\alpha}{4}\right),
$$

we obtain the left-hand member of (42) in the form

$$
\begin{aligned}
{\left[\phi_{2}^{4}\left(x+\frac{\alpha}{4}\right)+\right.} & \left.\psi_{2}^{4}\left(x+\frac{\alpha}{4}\right)\right] \cdot\left[\phi_{2}^{4}\left(y+\frac{\alpha}{4}\right)+\psi_{2}^{4}\left(y+\frac{\alpha}{4}\right)\right] \\
& -4 \phi_{2}^{2}\left(x+\frac{\alpha}{4}\right) \psi_{2}^{2}\left(x+\frac{\alpha}{4}\right) \phi_{2}^{2}\left(y+\frac{\alpha}{4}\right) \psi_{2}^{2}\left(y+\frac{\alpha}{4}\right) .
\end{aligned}
$$

Now with the help of the third of relations (36) we have

$$
\begin{aligned}
\phi_{2}^{4}\left(\frac{\alpha}{4}\right)\left[\phi_{2}^{4}\left(x+\frac{\alpha}{4}\right)+\psi_{2}^{4}\left(x+\frac{\alpha}{4}\right)\right] & \\
= & {\left[\phi_{2}^{2}\left(x+\frac{\alpha}{4}\right) \psi_{2}^{2}\left(\frac{\alpha}{4}\right)-\psi_{2}^{2}\left(x+\frac{\alpha}{4}\right) \phi_{2}^{2}\left(\frac{\alpha}{4}\right)\right]^{2} } \\
& +2 \phi_{2}^{4}\left(\frac{\alpha}{4}\right) \phi_{2}^{2}\left(x+\frac{\alpha}{4}\right) \psi_{2}^{2}\left(x+\frac{\alpha}{4}\right) \\
= & \phi_{2}^{2}\left(x+\frac{\alpha}{2}\right) \phi_{2}^{2}(x)+2 \phi_{2}^{4}\left(\frac{\alpha}{4}\right) \phi_{2}^{2}\left(x+\frac{\alpha}{4}\right) \psi_{2}^{2}\left(x+\frac{\alpha}{4}\right)
\end{aligned}
$$

and also$$
\phi_{2}\left(x+\frac{\alpha}{4}\right) \psi_{2}\left(x+\frac{\alpha}{4}\right)=-\phi_{2}\left(x+\frac{\alpha}{4}\right) \phi_{2}\left(x-\frac{\alpha}{4}\right)
$$$$
=\phi_{2}^{2}\left(\frac{\alpha}{4}\right)\left[\psi_{2}^{2}(x)-\phi_{2}^{2}(x)\right]=\phi_{2}^{2}\left(\frac{\alpha}{4}\right)\left[\phi_{2}^{2}\left(x+\frac{\alpha}{2}\right)-\phi_{2}^{2}(x)\right] .
$$

By means of these two equations the argument $x+\alpha / 4$ and similarly $y+\alpha / 4$ may be eliminated from (43), whereby it becomes identical with the righthand member of (42). Thus (40) becomes an identity when $k_{i}^{2}$ is either root of (41), and consequently the second of equation $(A)$ is satisfied by (35) after it has been squared.

It remains to show that the last mentioned equation is also satisfied before being squared. Now this is obvious for $x=y=0$, and when $x, y$ are continuously varied, its two members must continue to remain equal so long as $x, y$ do not pass through values which make the left-hand member vanish; i. e., exceptional values for which either 


$$
\psi_{i}(x+y)=0 \text { or } \quad \psi_{i}(x-y)=0 .
$$

For fixed $x$ or for fixed $y$ the values of $y$ and $x$ respectively which satisfy either equation form a set of isolated values.* Let $x^{\prime}, y^{\prime}$ be any pair of values for which neither $\psi_{i}\left(x^{\prime}+y^{\prime}\right)=0$ nor $\psi_{i}\left(x^{\prime}-y^{\prime}\right)=0$. Then by continuous variation it is possible to pass from $x=0, y=0$ to $x=x^{\prime}, y=y^{\prime}$ without passing through exceptional values of $x, y$. For first, $x$ may be held equal to 0 (or a very small $\epsilon$ ) while $y$ is varied from 0 to $y^{\prime}$ in such manner as to avoid exceptional values, and then while $y$ remains at $y^{\prime}, x$ may be varied in like manner from 0 (or $\epsilon$ ) to $x^{\prime}$. The second of equations $(A)$ then holds for $x=x^{\prime}, y=y^{\prime}$ before being squared and hence it is always valid. We conclude therefore that for the two values of $k_{i}^{2}$ furnished by (41) the functions (35) will satisfy $(A)$.

It has thus been shown that with $\phi_{2}(x), \psi_{2}(x)$ we may ally two and only two solutions of form $\phi_{i}(x)=c_{i} \phi_{2}(x), \psi_{i}(x)$, to which the indices $i=1$, 3 will be assigned. Four allied functions are thereby connected, as in the theory of the Jacobi $\vartheta$-functions. From (38) and (41) we get

$$
1=k_{1}^{2} k_{3}^{2}=\psi_{1}^{2}\left(\frac{\alpha}{2}\right) \psi_{3}^{2}\left(\frac{\alpha}{2}\right)
$$

Then from (37) and (44) it follows that

$$
\psi_{1}^{2}(x) \equiv \frac{\psi_{3}^{2}\left(x+\frac{\alpha}{2}\right)}{\psi_{3}^{2}\left(\frac{\alpha}{2}\right)}, \quad \psi_{3}^{2}(x) \equiv \frac{\psi_{1}^{2}\left(x+\frac{\alpha}{2}\right)}{\psi_{1}^{2}\left(\frac{\alpha}{2}\right)} .
$$

Furthermore, since every $\psi(x)$ possesses zeros whose doubles are zeros of $\phi(x)$ and since $\psi_{1}(x), \psi_{3}(x)$ do not vanish at $\alpha / 2$, it follows that $\phi_{2}(x)$ must have two independent sets of zeros, $\pm n \alpha$ and $\pm n \alpha^{\prime}$. Then simultaneously the three allied $\psi_{i}(x)$ have each a doubly periodic set of zeros, $\psi_{2}(x)$ vanishing at $\alpha / 2$, a second at $\alpha^{\prime} / 2$, and the remaining one by (45) at $\left(\alpha+\alpha^{\prime}\right) / 2$.

\section{The SIMPly PERIOdic CASE}

While in general there are three allied solutions, an exception occurs when the two roots of the quadratic (41) are equal, namely when

$$
\phi_{2}\left(\frac{\alpha}{4}\right)= \pm \frac{1}{\sqrt{2}}, \quad \pm \frac{i}{\sqrt{2}}, \quad \pm \frac{i+1}{2} .
$$

\footnotetext{
* As $\phi_{i}(x), \psi_{i}(x)$ satisfy equations (2) when squared, the argument of $\S 1$ applies without change to show that the double of a root $\beta$ of $\psi_{i}(x)$ is a root of $\phi_{i}(x)$. As the roots of $\phi_{i}(x) \equiv c_{i} \phi_{2}(x)$ are isolated, the same must be true of the roots of $\psi_{i}(x)$.
} 
We have then $k_{i}^{2}=1, c_{i}=0$, and $\phi_{i}(x) \equiv 0(i=1,3)$. Thus two allied solutions degenerate, and by $\S 2$ the function

$$
\psi_{i}^{2}(x)=\phi_{2}^{2}(x)+\phi_{2}^{2}\left(x+\frac{\alpha}{2}\right)=\phi_{2}^{2}(x)+\psi_{2}^{2}(x) \quad(i=1,3)
$$

becomes merely an expression of form (11). By the removal of this expression as a common factor from $\phi_{2}^{2}(x), \psi_{2}^{2}(x)$, our last equation reduces to

$$
1=\phi^{2}(x)+\psi^{2}(x) \text {, }
$$

where the subscripts are now discarded as useless. The preceding equation for $\psi_{i}^{2}(x)$ shows that the factor removed was one which remains unaltered when $x$ is replaced by $x+\alpha / 2$. Its removal therefore in no wise affects the normalization (22) for $\phi_{2}(x)$ and $\psi_{2}(x)$ with respect to $\beta=\alpha / 2$, which accordingly survives for $\phi(x), \psi(x)$ in (47).

By means of (47) the form of our functional equations $(A)$ may be simplified. By elimination of $\psi^{2}$ they become

$$
\begin{aligned}
& \phi(x+y) \phi(x-y)=\phi^{2}(x)-\phi^{2}(y), \\
& \psi(x+y) \psi(x-y)=1-\phi^{2}(x)-\phi^{2}(y) .
\end{aligned}
$$

The last equation gives

$$
\psi(2 x)=1-2 \phi^{2}(x) .
$$

Then by combination of the preceding equations and the substitution of $x, y$ for $x+y, x-y$ we have

$$
\psi(x) \psi(y)-\phi(x) \phi(y)=\psi(x+y),
$$

whence it follows by the substitution of $x+\alpha / 2$ for $x$ and by use of (22) that

$$
\phi(x) \psi(y)+\phi(y) \psi(x)=\phi(x+y) .
$$

Thus for the special case before us $(A)$ may be replaced by the familiar equations $(D)$, but with a restriction to be immediately specified.

To obtain the general continuous solution of $(D)$ place

$$
f(x)=\psi(x) \pm i \phi(x) .
$$

Then $(D)$ gives the equation

$$
f(x+y)=f(x) f(y),
$$

of which the general solution $(f(x) \equiv 0$ excepted) is

Hence

$$
\begin{array}{cl}
f(u+i v) \equiv f(u) f(i v)=e^{d_{1} u+d_{2} v} . & \\
\psi(x)+i \phi(x)=e^{d_{1} u+d_{2} v} & (x=u+i v) \\
\psi(x)-i \phi(x)=e^{d_{3} u+d_{4} v} &
\end{array}
$$


and

$$
\begin{aligned}
& \phi(x)=\frac{1}{2 i}\left(e^{d_{1} u+d_{2} v}-e^{d_{3} u+d_{4} v}\right), \\
& \psi(x)=\frac{1}{2}\left(e^{d_{1} u+d_{2} v}+e^{d_{3} u+d_{4} v}\right)
\end{aligned}
$$

$$
(x=u+i v) \text {. }
$$

By substitution it will be found that $(D)$ is satisfied by (48) regardless of the values of the constants, and accordingly (48) furnishes the general solution of $(D)$.

The condition (47) requires in addition that we make $d_{3}=-d_{1}, d_{4}=-d_{2}$, which indeed expresses the necessary and sufficient condition that the functions (48) shall be the one odd and the other even. Then the familiar subtraction as well as addition formulas are fulfilled, and hence equations $(A)$. Thus in the simply periodic case the general solution of $(A)$, save for an arbitrary factor of form (11), is expressible in the form

$$
\begin{aligned}
& \phi(x)=\frac{1}{2 i}\left(e^{d_{1} u+d_{2} v}-e^{-\left(d_{1} u+d_{2} v\right)}\right), \\
& \psi(x)=\frac{1}{2}\left(e^{d_{1} u+d_{2} v}+e^{-\left(d_{1} u+d_{2} v\right)}\right),
\end{aligned}
$$

or as an "equivalent" solution.

The zeros of $\phi(x), \psi(x)$ lie at the points for which $e^{2 d_{1} u+2 d_{2} v}$ is equal to +1 or -1 respectively. Put $d_{1}=d_{1}^{\prime}+i d_{1}^{\prime \prime}, d_{2}=d_{2}^{\prime}+i d_{2}^{\prime \prime}$. Then unless $\Delta \equiv d_{1}^{\prime} d_{2}^{\prime \prime}-d_{2}^{\prime} d_{1}^{\prime \prime}=0$, there will be a line through the origin on which $\phi(x), \psi(x)$ possess each a simply periodic set of zeros which alternate with one another. An exception occurs for any solution having $\Delta=0$. Then there is a line through the origin along which $\phi(x)$ everywhere vanishes, while $\psi(x)$ does not vanish at all unless $d_{1}^{\prime}=d_{2}^{\prime}=0$. In this latter case a set of equidistant parallel lines exist along which $\phi(x)$ everywhere vanishes (cf. §3) while $\psi(x)$ vanishes along the lines half way between consecutive vanishing lines for $\phi(x)$.

In conclusion, it should be pointed out that not all the values given by (46) can be taken by $\phi_{2}(x)$. In fact, if we put $x=\alpha / 4$ in (47) we have

$$
\phi^{2}(\alpha / 4)=1 / 2
$$

since $\psi^{2}(\alpha / 4)=\phi^{2}(3 \alpha / 4)=\phi^{2}(\alpha / 4)$. Now the factor which was removed in passing from $\phi_{2}^{2}(x)$ to $\phi^{2}(x)$ was one of form (11) which had the property of remaining unaltered when $x$ was replaced by $x+\alpha / 2$ and hence, if

$$
\alpha=\alpha^{\prime}+i \alpha^{\prime \prime},
$$

it may be written under the form 


$$
e^{c_{1}\left(a^{\prime \prime} u^{2}-2 a^{\prime} a^{\prime \prime} u u^{\prime}+\alpha^{\prime 2} v^{2}\right)}
$$

For $u+i v=\alpha / 4$ this takes the value 1 . Hence only the first pair of values given in (46) is admissible for $\phi_{2}(x)$. The second set $\pm i / \sqrt{2}$ gives the value of $\phi(\alpha / 4)$ for certain equivalent solutions, while the third set is altogether to be excluded. We conclude therefore that the necessary and sufficient condition that a solution $\phi(x), \psi(x)$ shall have a simply periodic set of zeros is that the zeros of $\phi(x)$ shall be discrete (i. e., not form continuous lines) and that the normalized function $\phi_{2}(x) \equiv \phi(x) / \phi(\alpha / 2)$ shall have the value $\pm 1 / \sqrt{2}$ for $x=\alpha / 4$, when $\alpha$ is the primitive zero of $\phi(x)$ with respect to which it is normalized. The conditions are indeed necessary, for otherwise, as we have seen, there will be a system of three allied solutions, each with a doubly periodic system of zeros.

\section{The doubly PeRIodic case}

In $\$ 4$ the normalization of the solution was made with reference to any primitive root $\alpha$ of $\phi(x)$. In the ordinary theory of trigonometric or $\vartheta$ functions this root has the position $\pi$. To this position $\alpha$ may be brought by an appropriate affine transformation of the $x$-plane with real coefficients:

$$
\begin{aligned}
& u^{\prime}=a u+b v, \\
& v^{\prime}=c u+d v .
\end{aligned}
$$

$$
\left(\begin{array}{c}
x=u+i v \\
x^{\prime}=u^{\prime}+i v^{\prime}
\end{array}\right)
$$

Any such transformation converts any four arguments $x, y, x-y, x+y$ into a like quadruple $x^{\prime}, y^{\prime}, x^{\prime}-y^{\prime}, x^{\prime}+y^{\prime}$, and hence the functions $\phi(x)$, $\psi(x)$ after transformation satisfy $(A)$. By such transformation there is connected with any one solution a family of essentially equivalent solutions which are merely the continuous distortion of that solution through affine transformations of the $x$-plane which leave the origin unaltered. In particular, with any analytic solution there is thus connected a family of non-analytic solutions. There exist, however, non-analytic solutions of $(A)$ which are not connected in this manner with analytic solutions, as for example those of $\S 3$. In the simply periodic case of $\S 7$ it is easy to verify that when $\Delta \neq 0$, the solution is reducible by a real affine transformation (50) to the form

$$
\phi(x)=\sin \mu x, \quad \psi(x)=\cos \mu x,
$$

in which $\mu$ is a constant, real or complex. Except for a factor (11) all solutions of $\S 7$ which do not fall under $\S 3$ are therefore either analytic or the affine distortions of analytic solutions. It seems probable that the like is true also for the doubly periodic case.

In considering henceforth the doubly periodic case we will suppose a primi- 
tive pair of zeros brought by an affine transformation to positions $\alpha=\pi$, $2 \beta=i \log q$ on the real and imaginary axis respectively. In effecting the normalization of $\phi(x)$ with respect to $\alpha$ in $\S 4$ only a part of the arbitrary factor (11) was employed and we still have the liberty to modify $\phi(x), \psi(x)$ by a factor $e^{c_{3} v^{2}}$, which in no wise disturbs that normalization if $\alpha$ is real. Consider a solution $\phi(x), \psi(x)$ for which $\beta$ is a root of $\psi(x)$. By the introduction of said factor the first equation of (20) becomes

$$
e^{c_{3}\left(2 \beta^{\prime \prime v}+\beta^{\prime \prime 2}\right)} \phi(x+\beta)=b e^{b_{1} u+b_{2} v} \psi(x) \quad\left(\beta=\beta^{\prime}+i \beta^{\prime \prime}\right),
$$

and hence by proper choice of $c_{3}$ we may bring (20) to the form

$$
\begin{aligned}
& \phi(x+\beta)=b^{\prime} e^{b_{1} x} \psi(x), \\
& \psi(x+\beta)= \pm b^{\prime} e^{b_{1} x} \phi(x) .
\end{aligned}
$$

Here $b^{\prime}, b_{1}$ must be conditioned by the requirement that the two equations shall be consistent for $x=-\beta / 2$, which gives

$$
\pm{b^{\prime}}^{2} e^{-i b_{1} \log q / 2}=-1 \text {. }
$$

Replacing $x$ in the first equation of (51) by $x+\alpha \equiv x+\pi$ and then comparing it with the same equation before replacement, we obtain with the aid of (18) or (19) either $e^{l_{1} \pi}=+1$ or $e^{b_{1} \pi}=-1$, and therefore $b_{1}=n i$, where $n$ is some integer, positive, negative, or zero. Equations (51) now take the form:

or

$$
\begin{aligned}
& \phi\left(x+\frac{i \log q}{2}\right)= \pm \frac{e^{i n x}}{q^{n / 4}} \psi(x), \\
& \psi\left(x+\frac{i \log q}{2}\right)=\mp \frac{e^{i n x}}{q^{n / 4}} \phi(x),
\end{aligned}
$$

$$
\begin{aligned}
& \phi\left(x+\frac{i \log q}{2}\right)= \pm \frac{i e^{i n x}}{q^{n / 4}} \psi(x), \\
& \psi\left(x+\frac{i \log q}{2}\right)= \pm \frac{i e^{i n x}}{q^{n / 4}} \phi(x) .
\end{aligned}
$$

The four alternatives offered by these two sets of formulas are realized simultaneously by equivalent solutions of $(A)$.

When the functions are analytic, we cannot have $n=0$, for then they would have $2 \pi$ and $4 i \log q$ as periods, which is impossible for analytic functions everywhere finite. If $n \neq 0$, the form of our formulas may be simplified to accord with that found in the theory of the $\vartheta$-functions. Replace $x$ by $x / n$, and $\phi(x / n), \psi(x / n), q^{n}$ by $\phi(x), \psi(x), q$. Formulas (52) and (53) result with $n$ now equal to 1 . 
It has been already noted that the allied solutions are simultaneously normalized. In particular, equations (18) hold for $\phi_{2}(x), \psi_{2}(x)$ and (19) for $\phi_{i}(x), \psi_{i}(x),(i=1,3)$.

The theory of the $\vartheta$-functions furnishes the following allied solutions of $(A)$,

$$
\begin{aligned}
\text { (II) } \phi_{2}(x) & =\frac{\vartheta_{1}(x)}{\vartheta_{1}\left(\frac{\pi}{2}\right)=\vartheta_{2}(0)} \equiv \sqrt[4]{\left(e_{1}-e_{3}\right)\left(e_{1}-e_{2}\right)} e^{-2 \eta_{1} \omega_{1} x^{2}} \sigma(u), \\
\psi_{2}(x) & =\frac{\vartheta_{2}(x)}{\vartheta_{2}(0)} \equiv e^{-2 \eta_{1} \omega_{1} x^{2}} \sigma_{1}(u), \\
\text { (I) } \quad \phi_{1}(x) & =\frac{\vartheta_{1}(x)}{\vartheta(0)} \equiv \sqrt[4]{\left(e_{3}-e_{1}\right)\left(e_{3}-e_{2}\right)} e^{-2 \eta_{1} \omega_{1} x^{2}} \sigma(u), \\
\psi_{1}(x) & =\frac{\vartheta(x)}{\vartheta(0)} \equiv e^{-2 \eta_{1} \omega_{1} x^{2}} \sigma_{3}(u), \\
\text { (III) } \phi_{3}(x) & =\frac{\vartheta_{1}(x)}{\sqrt{i} \vartheta_{3}(0)} \equiv \sqrt[4]{\left(e_{2}-e_{1}\right)\left(e_{2}-e_{3}\right)} e^{-2 \eta_{1} \omega_{1} x^{2}} \sigma(u), \\
\psi_{3}(x) & =\frac{\vartheta_{3}(x)}{\vartheta_{3}(0) \equiv \vartheta\left(\frac{\pi}{2}\right)} \equiv e^{-2 \eta_{1} \omega_{1} x^{2}} \sigma_{2}(u),
\end{aligned}
$$

where $u=2 \omega_{1} x$. The substitution of these pairs of functions in $(A)$ gives, in fact, six fundamental relations between these functions. Instead of regarding these functions as an application of our theory, we may conceive I-III as defining four $\vartheta$-functions in terms of our allied solutions. For the constants of our preceding theory we have the equivalents

$$
\begin{gathered}
c_{1}=\frac{\vartheta_{2}(0)}{\vartheta(0)}, \quad c_{3}=\frac{\vartheta_{2}(0)}{\sqrt{i} \vartheta_{3}(0)}, \quad k_{1}^{2}=\psi_{1}^{2}\left(\frac{\pi}{2}\right)=\frac{\vartheta^{2}\left(\frac{\pi}{2}\right)}{\vartheta^{2}(0)}=\frac{\vartheta_{3}^{2}(0)}{\vartheta^{2}(0)}, \\
k_{3}^{2}=\psi_{3}^{2}\left(\frac{\pi}{2}\right)=\frac{\vartheta_{3}^{2}\left(\frac{\pi}{2}\right)}{\vartheta_{3}^{2}(0)} .
\end{gathered}
$$

Then by (44) we get

$$
\vartheta_{3}^{2}\left(\frac{\pi}{2}\right)=\vartheta^{2}(0)
$$

The application of (39) gives then the fundamental relation:

$$
\vartheta_{3}^{4}(0)=\vartheta^{4}(0)+\vartheta_{2}^{4}(0) .
$$

To make the relations between the $\vartheta$-functions thus defined identical with the ordinary $\vartheta$-relations it will be necessary to make a proper selection from 
the three allied sets of equivalent solutions. The choice of $\phi_{2}(x), \psi_{2}(x)$ has already been made definite by the requirement that they shall be equal to 1 at $x=\pi / 2$ and $x=0$ respectively. For $\phi_{1}(x), \psi_{1}(x)$ select the allied solution which has a zero at $i \log q / 2$ and which satisfies equations of form (53) with the lower or negative signs in the right-hand members. As the remaining pair of allied functions take in accord with (33)

$$
\phi_{3}(x)=\frac{\phi_{1}(x)}{\sqrt{i} \psi_{1}\left(\frac{\pi}{2}\right)}, \quad \psi_{3}(x)=\frac{\psi_{1}\left(x+\frac{\pi}{2}\right)}{\psi_{1}\left(\frac{\pi}{2}\right)}
$$

In consequence of the definitions I-III and our selection of allied functions we have

$$
\begin{aligned}
\vartheta_{1}\left(x+\frac{\pi}{2}\right) & =\vartheta_{2}(x), \\
\vartheta_{2}\left(x+\frac{\pi}{2}\right) & =-\vartheta_{1}(x), \\
\vartheta_{3}\left(x+\frac{\pi}{2}\right) & =\vartheta(x), \\
\vartheta\left(x+\frac{\pi}{2}\right) & =\vartheta_{3}(x), \\
\vartheta_{1}\left(x+\frac{i \log q}{2}\right) & =-i q^{-n / 4} e^{i n x} \vartheta(x), \\
\vartheta\left(x+\frac{i \log q}{2}\right) & =-i q^{-n / 4} e^{i n x} \vartheta_{1}(x)
\end{aligned}
$$

where $n=1$ when the $\phi, \psi$ are analytic. Then by substitution of $x+\pi / 2$ for $x$ in the last two equations we get for $n=1$

$$
\begin{aligned}
& \vartheta_{2}\left(x+\frac{i \log q}{2}\right)=q^{-1 / 4} e^{i x} \vartheta_{3}(x), \\
& \vartheta_{3}\left(x+\frac{i \log q}{2}\right)=q^{-1 / 4} e^{i x} \vartheta_{2}(x)
\end{aligned}
$$

For $i=1,3$ equation (37) gives

$$
\begin{aligned}
& \vartheta_{2}^{2}(0) \vartheta^{2}(x)=\vartheta^{2}(0) \vartheta_{2}^{2}(x)+\vartheta_{3}^{2}(0) \vartheta_{1}^{2}(x) \\
& \vartheta_{2}^{2}(0) \vartheta_{3}^{2}(x)=\vartheta_{3}^{2}(0) \vartheta_{2}^{2}(x)+\vartheta^{2}(0) \vartheta_{1}^{2}(x)
\end{aligned}
$$

The substitution of (II) in (25) gives 


$$
\vartheta(0) \vartheta_{2}(0) \vartheta_{3}(0) \vartheta_{1}(2 x)=2 \vartheta_{1}(x) \vartheta(x) \vartheta_{2}(x) \vartheta_{3}(x),
$$

while from (4) we have

$$
\vartheta^{3}(0) \vartheta(2 x)=\vartheta^{4}(x)-\vartheta_{1}^{4}(x)
$$

with corresponding formulas for $\vartheta_{2}(x)$ and $\vartheta_{3}(x)$.

These formulas apply irrespective of the analytic or non-analytic character of the allied solutions. For differentiation formulas see the close of $\S 9$.

\section{A Fundamental Lemma}

Formulas (25) and (27) furnish the fundamental limit

$$
\operatorname{Lim}_{x=0} \frac{\phi(2 x)}{\phi(x)}=2
$$

and hence also

$$
\operatorname{Lim}_{x=0} \frac{R(2 x)}{R(x)}=2 \text {. }
$$

The proof of these formulas was based on the existence of a root of $\phi(x)$ not at the origin, which was established on the hypothesis that $\phi(x), \psi(x)$ exist over the complex plane. In the investigation of the real solutions the limit (59) is fundamental, and it will therefore be necessary to establish it without assuming the existence of the functions exterior to the real axis.

For this purpose replace $x, y$ in $(B)$ first by $x+a, x-a$, then by $x / 2+a$, $x / 2-a$, and divide the first resulting equation by the second. We obtain

$$
\frac{R(2 x)}{R(x)}=\frac{R^{2}(x+a)-R^{2}(x-a)}{R^{2}\left(\frac{x}{2}+a\right)-R^{2}\left(\frac{x}{2}-a\right)} \cdot \frac{1-R^{2}\left(\frac{x}{2}+a\right) R^{2}\left(\frac{x}{2}-a\right)}{1-R^{2}(x+a) R^{2}(x-a)}
$$

The limit of the second fraction on the right for $x=0$ is 1 , if $a$ is any point for which $R(a)$ is finite. Now by $(B)$ we have

and therefore

$$
\pm R(x \pm a) R(a)=\frac{R^{2}\left(\frac{x}{2} \pm a\right)-R^{2}\left(\frac{x}{2}\right)}{1-R^{2}\left(\frac{x}{2} \pm a\right) R^{2}\left(\frac{x}{2}\right)}
$$

$$
\begin{aligned}
& \frac{R^{2}(x+a)-R^{2}(x-a)}{R^{2}(x / 2+a)-R^{2}(x / 2-a)} \\
&=\frac{1}{R^{2}(a)} \cdot \frac{R^{2}\left(\frac{x}{2}+a\right)+R^{2}\left(\frac{x}{2}-a\right)-R^{2}\left(\frac{x}{2}\right)[\quad]}{1-R^{2}\left(\frac{x}{2}\right)[]},
\end{aligned}
$$


in which the square brackets abbreviate expressions which need not be written down. If this value is substituted in the first fraction on the right of (60) and the limit is then sought for $x=0$, we immediately obtain the limit (59) and hence also (58).

A consequence of (58) is that the zeros of $\phi(x)$ must all be simple when it is analytic. For, the expansion

$$
\phi(x)=A_{0} x+A_{1} x^{2}+\cdots
$$

is consistent with (58) only if $A_{0} \neq 0$. The zero at the origin is therefore of first order, whence it follows by (5) and (6) that all other zeros of $\phi(x)$ and $\psi(x)$ must be of first order.

Regarding the differentiability of $R(x)$ it may be noted that it is differentiable over the entire axis or plane on which its existence is assumed, provided that it is differentiable at the origin. For from $(B)$ we have

$$
\frac{R(x)-R(y)}{x-y}=\frac{R(x+y)\left[1-R^{2}(x) R^{2}(y)\right]}{R(x)+R(y)} \cdot \frac{R(x-y)}{x-y} .
$$

If now $y$ approaches $x$ as a limit, this gives

$$
\frac{d R(x)}{d x}=\frac{R(2 x)\left[1-R^{4}(x)\right]}{2 R(x)} \cdot\left(\frac{d R(x)}{d x}\right)_{x=0} .
$$

Application to solution I gives with the aid of (56) and (57)

$$
\frac{d\left(\frac{\vartheta_{1}(x)}{\vartheta x}\right)}{d x}=\frac{\vartheta^{2}(0) \vartheta_{2}(x) \vartheta_{3}(x)}{\vartheta_{2}(0) \vartheta_{3}(0) \vartheta^{2}(x)} \cdot\left(\frac{d \frac{\vartheta_{1}(x)}{\vartheta(x)}}{d x}\right)_{x=0} .
$$

Similar formulas hold for the derivatives of $\vartheta_{1}(x) / \vartheta_{2}(x)$ and $\vartheta_{1}(x) / \vartheta_{3}(x)$.

\section{Determination of the Real solutions}

In determining the real solutions of $(A)$ we shall first seek real solutions of

$$
R(x+y) R(x-y)=\frac{R^{2}(x)-R^{2}(y)}{1-R^{2}(x) R^{2}(y)}
$$

Independently of $(A)$ this equation may be considered on the hypothesis that $R(x)$ is one-valued and continuous on the real axis or over the complex plane, except at isolated points at which it becomes infinite. When $R(x)=\infty$, the value to be attributed to the right-hand member is, of course, the limiting value $-1 / R^{2}(y)$. This member loses significance only when

$$
R^{2}(x)=R^{2}(y)= \pm 1 \text {. }
$$

When for particular values of $x$ or $y$ either $R(x+y)$ or $R(x-y)$ becomes 
infinite, their product must tend to a limiting value equal to that taken by the right-hand member of $(B)$.

By simple considerations it appears that $R(x)$ is an odd function which vanishes at the origin.

If $R^{2}(x)= \pm 1$ for $x=\gamma$, we have by $(B)$

$$
R(x+\gamma) R(x-\gamma)= \pm 1 .
$$

Hence when we place $y=x+2 \gamma$ in $(B)$, it follows that $2 \gamma$ is an infinity of $R(x)$. The points at which $R^{2}(x)= \pm 1$ are therefore isolated.

Suppose $x=\alpha$ to be a root of $R(x)$ and put $y=x+\alpha$ in $(B)$. We get

$$
R^{2}(x+\alpha)=R^{2}(x)
$$

except possibly for values of $x$ which make $R(2 x+\alpha)=\infty$. But then by modifying slightly such an exceptional value and passing in (61) to this value of $x$ as limit, it follows that this equation is still valid. Hence $\alpha$ is a period of $R^{2}(x)$. In consequence the zeros of $R(x)$ on the real axis or any line through the origin must be isolated, unless $R(x)$ vanishes identically along the line.

Similarly when $x=\beta$ is an infinity of $R(x)$, put $y=x+\beta$ in $(B)$. We get

$$
R^{2}(x+\beta)=\frac{1}{R^{2}(x)},
$$

the equation holding by considerations of continuity even if $R(x+2 \beta)=0$. Thus $2 \beta$ must be a period and a zero of $R^{2}(x)$.

Let $\beta$ be any infinity so situated that no infinity lies within the segment $(0, \beta)$. The segment cannot contain a zero $x=\alpha$, for $\alpha$ is a period of $R^{2}(x)$, and hence there would be an infinity within the segment. For any particular $x$ within the segment we have either

$$
R(x+\beta)=\frac{1}{R(x)} \quad \text { or } \quad R(x+\beta)=-\frac{1}{R(x)} .
$$

The sign of the right-hand member cannot change within the segment because of the continuity of the function. Furthermore, for $y=\beta$ equation $(B)$ gives

$$
R(x+\beta) R(x-\beta)=\frac{1}{R^{2}(x)},
$$

from which it follows that the same sign must continue to hold throughout the entire line joining $x=0$ and $x=\beta$. When $R(x)$ is supposed to exist over the entire complex plane the same conclusion holds over the plane because $R(x)$ is continuous except at isolated poles. Thus for given $\beta$ one or the other of equations (62) holds independently of $x$. 
Let $\alpha$ denote a primitive root of $R(x)$. For any particular $x$ equation (61) gives either

$$
R(x+\alpha)=R(x) \quad \text { or } \quad R(x+\alpha)=-R(x) .
$$

If $\alpha / 2$ is an infinity of $R(x)$, the former equation is in force because of (62). If $\alpha / 2$ is not an infinity, then we can apply reasoning exactly like that of the preceding paragraph to show that for each $\alpha$ one or the other of the two equations (63) is universally applicable.

Seek now to determine the real solutions of $(B)$ by arbitrarily assuming values for $R(x)$ at two initial points $x^{\prime}, 2 x^{\prime}$. Unless $R(x)$ is constant and therefore everywhere 0 , we can take an initial $x^{\prime}$ for which $R^{2}\left(x^{\prime}\right) \neq 0,1, \infty$. If necessary, make a very small alteration in $x^{\prime}$ so that also $R\left(2 x^{\prime}\right) \neq \infty$. Then by putting $x=x^{\prime}$ and successively $y=2 x^{\prime}, 3 x^{\prime}, \cdots$ in $(B)$ we determine the sequence $R\left[(n+1) x^{\prime}\right],(n=2,3, \cdots)$, unless we come to a value $n=n^{\prime}$ which makes $R^{2}\left[\left(n^{\prime}+1\right) x^{\prime}\right]$ equal to $R^{2}\left(x^{\prime}\right)$ or to $1 / R^{2}\left(x^{\prime}\right)$. When this happens, $R\left(n^{\prime} x^{\prime}\right)$ is 0 or $\infty$ so that one of the equations (62) or (63) respectively comes into play and $n^{\prime} x^{\prime}$ is a period or half-period. A comparison of $R\left(n^{\prime}+1\right) x^{\prime}$ with $R\left(x^{\prime}\right)$ will show which of the two corresponding equations is to be selected. The equation then serves to carry on the sequence of values for $R\left(n x^{\prime}\right)$ indefinitely. Thus $R(x)$ is determined uniquely over the point set $\left\{n x^{\prime}\right\}$ in terms of the two initial values.

When $y$ is successively placed equal to $2 x$ and $3 x$ in $(B)$, two equations are obtained between which $R(3 x)$ may be eliminated. If $x$ in the resulting equation is replaced by $x^{\prime} / 2$, the equation may be written:

$$
R^{4}\left(\frac{x^{\prime}}{2}\right)-R^{2}\left(\frac{x^{\prime}}{2}\right)\left[\frac{2+\frac{R\left(2 x^{\prime}\right)}{R\left(x^{\prime}\right)}\left(1-R^{4}\left(x^{\prime}\right)\right)}{R^{2}\left(x^{\prime}\right)}\right]+1=0
$$

which serves to determine $R\left(x^{\prime} / 2\right)$. This equation must not admit as root $R^{2}\left(x^{\prime} / 2\right)= \pm 1$, for then on replacing $x^{\prime}$ by $x^{\prime} / 2^{m}(m=1,2, \cdots)$ we would obtain $R^{2}\left(x^{\prime} / 2^{m}\right)= \pm 1$ in conflict with the limit (59). To exclude these values our initial values $R\left(x^{\prime}\right), R\left(2 x^{\prime}\right)$ must now be subjected to the restriction that they shall not be connected by the relation

$$
R\left(2 x^{\prime}\right)=\frac{-2 R\left(x^{\prime}\right)}{1 \pm R^{2}\left(x^{\prime}\right)}
$$

When the initial values satisfy the equation

we obtain from (64)

$$
R\left(2 x^{\prime}\right)=\frac{2 R\left(x^{\prime}\right)}{1 \pm R^{2}\left(x^{\prime}\right)}
$$




$$
R^{2}\left(x^{\prime}\right)=\left[\frac{2 R\left(\frac{x^{\prime}}{2}\right)}{1 \pm R^{2}\left(\frac{x^{\prime}}{2}\right)}\right]^{2},
$$

and only then. When therefore the initial values satisfy either relation of (66), they must continue to satisfy the same relation when $x^{\prime}$ is replaced by $x^{\prime} / 2^{m}$, since otherwise the impossible relation (65) will be encountered. With this understanding (65) will be avoided when the argument is successively halved.

Select now some one of the roots of (64) as the value of $R\left(x^{\prime} / 2\right)$ and do the same when $x^{\prime}$ is successively replaced by $x^{\prime} / 2^{m}(m=1,2, \cdots)$. The choice of root is ultimately restricted by (59). Now the roots of (64) form two pairs, those of either pair being the negative of one another and the reciprocal of those of the other. Since $R(0)=0$, the choice of roots is ultimately restricted to the smaller pair, and finally for sufficiently large $m$ to a definite one of this pair because of (59). Let this be for $m \geqq N$. Then if the initial $x^{\prime}$ is replaced by $x^{\prime} / 2^{N}$, as we shall now suppose, and if the notation $x^{\prime}$ is retained for the new initial point, the determination of $R\left(x^{\prime} / 2^{m}\right)(m=1$, $2, \cdots)$ will be unique.

By combination of preceding results $R(x)$ is determined uniquely in terms of the initial values over the dense set of points $\left\{n x^{\prime} / 2^{m}\right\},(n, m,=1$, $2, \cdots)$. The result can be extended by the equation $R(-x)=-R(x)$ to the other half of the line connecting $x^{\prime}$ with the origin. It remains for subsequent consideration whether the set of values thus obtained is consistent with the continuity requirement for $R(x)$.

As yet the requirement of reality has not been imposed. Suppose henceforth the initial points and values to be real. Then $R\left(n x^{\prime}\right)$ is real, and it remains to examine under what conditions $R\left(x^{\prime} / 2^{m}\right)$ will be real. Clearly we obtain positive values for $R^{2}\left(x^{\prime} / 2\right)$ from (64) if and only if

$$
\frac{R\left(2 x^{\prime}\right)}{R\left(x^{\prime}\right)} \cdot\left[1-R^{4}\left(x^{\prime}\right)\right]+2 \geqq 2 R\left(x^{\prime}\right)^{2} .
$$

When $\left|R\left(x^{\prime}\right)\right|>1$, this may be written

Thus the ratio

$$
-\frac{R\left(2 x^{\prime}\right)}{R\left(x^{\prime}\right)} \geqq \frac{2}{1+R^{2}\left(x^{\prime}\right)} \quad\left(\left|R\left(x^{\prime}\right)\right|>1\right) .
$$

$$
q\left(x^{\prime}\right) \equiv \frac{R\left(2 x^{\prime}\right)}{R\left(x^{\prime}\right)}
$$

should be negative for $\left|R\left(x^{\prime}\right)\right|>1$.

If $\left|R\left(x^{\prime}\right)\right|<1$, we have 


$$
q\left(x^{\prime}\right) \geqq-\frac{2}{1+R^{2}\left(x^{\prime}\right)}
$$

Suppose henceforth that the initial values meet these conditions and seek whether they continue to fulfill them when $x^{\prime}$ is replaced by $x^{\prime} / 2$. Three cases will be distinguished, which with the transition cases cover all possibilities for the initial values.

Case 1.

$$
\left|R\left(x^{\prime}\right)\right|<1, \quad\left|q\left(x^{\prime}\right)\right|<\frac{2}{1+R^{2}\left(x^{\prime}\right)} .
$$

If $(64)$ is written

$$
2+q\left(x^{\prime}\right)\left[1-R^{4}\left(x^{\prime}\right)\right]=q^{2}\left(\frac{x^{\prime}}{2}\right)\left[1+R^{4}\left(\frac{x^{\prime}}{2}\right)\right]
$$

and is then combined with the second of the inequalities, we obtain

$$
q^{2}\left(\frac{x^{\prime}}{2}\right)\left[1+R^{4}\left(\frac{x^{\prime}}{2}\right)\right]<4-2 R^{2}\left(\frac{x^{\prime}}{2}\right) q^{2}\left(\frac{x^{\prime}}{2}\right),
$$

whence follows

$$
\left|q\left(\frac{x^{\prime}}{2}\right)\right|<\frac{2}{1+R^{2}\left(\frac{x^{\prime}}{2}\right)} .
$$

Moreover, we must select for $R\left(x^{\prime} / 2\right)$ a root of (64) having an absolute value less than 1 , since the condition (67) cannot be met when $x^{\prime}$ is replaced by $x^{\prime} / 2$. With this understanding both inequalities of case 1 will continue to be fulfilled when $x^{\prime}$ is replaced by $x^{\prime} / 2$.

Case 2.

$$
\frac{2}{\left|1-R^{2}\left(x^{\prime}\right)\right|}>\left|q\left(x^{\prime}\right)\right|>\frac{2}{\left|1+R^{2}\left(x^{\prime}\right)\right|} .
$$

First suppose $\left|R\left(x^{\prime}\right)\right|<1$. Then $q\left(x^{\prime}\right)$ is positive, as otherwise (68) is not fulfilled. We can now combine either the first or second inequality of case 2 with (69) in the same manner as in case 1 and thereby obtain like inequalities with $x^{\prime} / 2$ in place of $x^{\prime}$.

Next suppose $\left|R\left(x^{\prime}\right)\right|>1$. Then $q\left(x^{\prime}\right)$ is negative, and (69) may be written:

$$
2+\left|q\left(x^{\prime}\right)\right| \cdot\left[R^{4}\left(x^{\prime}\right)-1\right]=q^{2}\left(\frac{x^{\prime}}{2}\right)\left[1+R^{4}\left(\frac{x^{\prime}}{2}\right)\right] .
$$

The combination of this with the first inequality of case 2 gives in the same manner as before

$$
\left|q\left(\frac{x^{\prime}}{2}\right)\right|<\frac{2}{\left|1-R^{2}\left(\frac{x^{\prime}}{2}\right)\right|} .
$$


The other desired inequality for $\left|q\left(x^{\prime} / 2\right)\right|$ is obtained immediately by addition of

and

$$
2 q^{2}\left(\frac{x^{\prime}}{2}\right) R^{2}\left(\frac{x^{\prime}}{2}\right) \equiv 2 R^{2}\left(x^{\prime}\right)>2
$$

$$
q^{2}\left(\frac{x^{\prime}}{2}\right)\left[1+R^{4}\left(\frac{x^{\prime}}{2}\right)\right]>2 q^{2}\left(\frac{x^{\prime}}{2}\right) R^{2}\left(\frac{x^{\prime}}{2}\right)>2 .
$$

Thus the inequalities characteristic of case 2 continue to be satisfied when $x^{\prime}$ is replaced by $x^{\prime} / 2$.

The choice of root for $R\left(x^{\prime} / 2\right)$ in (64) is restricted by the requirement that $q\left(x^{\prime} / 2\right)$ shall be positive or negative according as $\left|R\left(x^{\prime} / 2\right)\right|$ is less or greater than 1 .

Case 3.

$$
\left|q\left(x^{\prime}\right)\right|>\frac{2}{\left|1-R^{2}\left(x^{\prime}\right)\right|} .
$$

This case may be handled in the same way as case 2 .

In addition to these cases we have the following transition cases:

Case 4.

Case 5.

$$
q\left(x^{\prime}\right)=\frac{2}{1+R^{2}\left(x^{\prime}\right)} .
$$

$$
q\left(x^{\prime}\right)=\frac{2}{1-R^{2}\left(x^{\prime}\right)} .
$$

As already pointed out, these conditions are conserved when $x^{\prime}$ is replaced by $x^{\prime} / 2$ and they are realized by the hyperbolic and trigonometric tangents of $c x$.

The conditions characteristic of the several cases have thus been shown to be conserved when $x^{\prime}$ is replaced by $x^{\prime} / 2^{m}$, and conversely they must be conserved when $x^{\prime}$ is replaced by $2^{m} x^{\prime}$. We will now prove that cases 1-3 for given initial values of $R\left(x^{\prime}\right), R\left(2 x^{\prime}\right)$ can be realized through our Jacobian quotients.

Consider first the solution (I). By (54)-(57) we have

$$
\begin{aligned}
R(2 x) \equiv \frac{\vartheta_{1}(2 x)}{\vartheta(2 x)} & =\frac{2 \frac{\vartheta_{1}(x)}{\vartheta(x)}}{1-\frac{\vartheta}{\vartheta^{2}(x)}} \cdot \frac{\vartheta(0) \vartheta(0) \vartheta_{2}(x) \vartheta_{3}(x)}{1+\frac{\vartheta_{1}^{2}(x)}{\vartheta^{2}(x)}} \\
& =\frac{2 R(x)}{1-R^{2}(x)} \cdot \frac{\sqrt{\left(1-\frac{R^{2}(x)}{k}\right)\left(1-k R^{2}(x)\right)}}{1+R^{2}(x)}
\end{aligned}
$$

in which we have now introduced the constants 


$$
\sqrt{k}=\frac{\vartheta_{2}(0)}{\vartheta_{3}(0)}, \quad \sqrt{k^{\prime}}=\frac{\vartheta(0)}{\vartheta_{3}(0)} \quad\left(k^{2}+k^{\prime 2}=1\right)
$$

which we suppose real. The real function

$$
R(x) \equiv \frac{\vartheta_{1}(x)}{\vartheta(x)}=\sqrt{k} \text { sn } v \quad\left(v=\vartheta_{3}^{2}(0) x\right)
$$

is periodic and takes all values between $\sqrt{k}$ and $-\sqrt{k}$. If $k$ is taken sufficiently large, there will be a set of points at which it takes an initial value $R\left(x^{\prime}\right)$ assigned under case 1 . By replacing $x$ by $c x$ any one of these points may be made to coincide with the initial point $x^{\prime}$. The position of $x^{\prime}$ on the real axis is therefore immaterial and need not be taken account of further in this and succeeding cases.

Put now $x=x^{\prime}$ in (70) and then let $k$ vary continuously from $\left|R\left(x^{\prime}\right)\right|$ to 1 . Then the radical in (70) varies from 0 to $1-R^{2}\left(x^{\prime}\right)$, and correspondingly $\left|q\left(x^{\prime}\right)\right|$ varies between the limits characteristic of case 1 . Hence $\left|R\left(2 x^{\prime}\right)\right|$ for given $R\left(x^{\prime}\right)$ can be made to take any assigned value consistent with case 1 by proper choice of $k$. At the same time $R\left(2 x^{\prime}\right)$ can be given a positive or negative sign at pleasure since upon replacing $x^{\prime}$ by $-\left(x^{\prime}+\pi\right)$ we have

$$
\begin{aligned}
R\left(-x^{\prime}-\pi\right) & =\frac{-\vartheta_{1}\left(x^{\prime}+\pi\right)}{\vartheta\left(x^{\prime}+\pi\right)}=\frac{\vartheta_{1}\left(x^{\prime}\right)}{\vartheta\left(x^{\prime}\right)}=R\left(x^{\prime}\right), \\
R\left(-2 x^{\prime}-2 \pi\right) & =-R\left(2 x^{\prime}\right) .
\end{aligned}
$$

Thus any initial values consistent with case 1 can be realized through our $\vartheta$-quotient.

The curve $y=\vartheta_{1}(x) / \vartheta(x)$ has the essential characteristics of the sine curve, ${ }^{*}$ consisting of a series of congruent arches alternately above and below the real axes which have their maximum amplitudes at the mid points of the arches. For a given value of $R\left(x^{\prime}\right)=\vartheta_{1}\left(x^{\prime}\right) / \vartheta\left(x^{\prime}\right)$ there are two sets of points $x^{\prime}$ periodically spaced, and for each point of one set $R\left(2 x^{\prime}\right)$ can be made to take the given initial value by varying $k$. Whether the point varies in position or is kept in statu quo by replacing $x$ by $c x$ and varying $c$ and $k$ simultaneously is immaterial. Now in case 1 when formula (64) was applied to obtain in succession the terms

$$
R\left(\frac{x^{\prime}}{2}\right), \quad R\left(\frac{x^{\prime}}{4}\right), \quad R\left(\frac{x^{\prime}}{8}\right), \quad \ldots
$$

just two values were possible for each member after the preceding ones had been fixed. These two values differed in sign but ultimately the sign in the sequence (71) becomes invariable because of the existence of the limit (59).

\footnotetext{
${ }^{*}$ Cf. Krause, Theorie der elliptischen Funktionen, pp. 68-69.
} 
To every possible succession of signs in (71) consistent with this requirement there corresponds a point of one of the two periodically spaced sets of points above. Precisely as in the case of the sine function when $\sin x^{\prime}$ is given, and for the same reason, every possible succession of signs in (71) is realizable by appropriately selecting $x^{\prime}$ from our two sets of points. It follows therefore that through the Jacobian quotient (I) case 1 can be completely realized, and no further restrictions upon (71) are necessary than those which have been already discovered.

For the solution II we obtain in like manner by placing

$$
\begin{aligned}
R(x) & =\frac{\vartheta_{1}(x)}{\vartheta_{2}(x)}, \\
R(2 x) & =\frac{2 R(x)}{1-R^{2}(x)} \cdot \frac{\frac{\vartheta_{2}(0) \vartheta_{2}(0) \vartheta(x) \vartheta_{3}(x)}{1+\vartheta_{3}(x)}}{1+\ell_{2}(x) \vartheta_{2}(x)} \\
& =\frac{2 R(x)}{1-R^{2}(x)} \cdot \frac{\sqrt{\left(1+k^{\prime} R^{2}(x)\right)\left(1+\frac{R^{2}(x)}{k^{\prime}}\right)}}{1+R^{2}(x)} .
\end{aligned}
$$

The zeros of $\vartheta_{1}(x)$ and $\vartheta_{2}(x)$ are simple and alternate with each other along the real axis. There must therefore be a set of points $x^{\prime}$ on the real axis for which our periodic quotient $R(x)$ assumes any assigned real value $R\left(x^{\prime}\right)$. If now for fixed $R(x)$ we allow $k^{\prime}$ to vary from 1 to 0 in the above equation, the radical will vary from $1+R^{2}(x)$ to $\infty$. The limits for $\left|q\left(x^{\prime}\right)\right|$ are therefore the same as in case 3 , and hence the initially preassigned value for $\left|R\left(2 x^{\prime}\right)\right|$ can be attained simultaneously with $R\left(x^{\prime}\right)$. Now in case 3 the sign of $R\left(2 x^{\prime}\right)$ must be such that $q\left(x^{\prime}\right)=R\left(2 x^{\prime}\right) / R\left(x^{\prime}\right)$ is positive when $\left|R\left(x^{\prime}\right)\right|<1$ and negative when $\left|R\left(x^{\prime}\right)\right|>1$. From the theory of the $\vartheta$ functions, it is easily verified that as $x$ increases from 0 to $\pi$, the quotient $R(x)=\vartheta_{1}(x) / \vartheta_{2}(x)$ increases from 0 through $R(\pi / 4)=1$ to $\infty$, then from $-\infty$ through $R(3 \pi / 4)=-1$ to 0 . Correspondingly $q(x)$ increases from 0 to $q(\pi / 4)=\infty$ and thence through negative values to $q(\pi / 2)=0$, while from $\pi / 2$ to 0 it passes through the same cycle of changes in opposite order. Hence the proper sign of $R\left(2 x^{\prime}\right)$ can be realized. Thus any initial values for $R\left(x^{\prime}\right)$ and $R\left(2 x^{\prime}\right)$ consistent with case 3 can be realized through the $\vartheta$-quotient (II).

It remains to see whether every possible sequence of values (71) resulting from the initial values through the use of (64) are realizable through our $\vartheta$-quotient. Now both in cases 2 and 3 there were two possible choices for any member $R\left(x^{\prime} / 2^{m}\right)$ of (71) after the preceding members have been fixed by use of (64), and these two differ in sign since the ratio $q\left(x^{\prime} / 2^{m}\right)$ of two 
successive members of $(71)$ is positive or negative according as $\left|R\left(x^{\prime} / 2^{m}\right)\right|$ is taken less or greater than 1 . The graph of $R(x)=\vartheta_{1}(x) / \vartheta_{2}(x)$ has the same general character as that of $\tan x$ since $R(x+\pi / 2)=-1 / R(x)$. For a given value of $R\left(x^{\prime}\right)$ there is a periodic set of points $x^{\prime} \pm n \pi$, for each of which the initial values can be realized by variation of $k$ in the manner already explained. As in the case of $\tan x^{\prime}$ and for the same reason all possible choices of sign in (71) can be realized subject to the condition that all terms shall ultimately be of same sign. Thus case 3 can be completely realized through the $\vartheta$-quotient (II) and with no further restriction upon (71) than has been already laid down.

The solution III makes $R(x)=\vartheta_{1}(x) / \sqrt{i} \vartheta_{3}(x)$, and correspondingly we have

$$
\begin{aligned}
R(2 x) & =\frac{2 R(x)}{1+R^{2}(x)} \cdot \frac{\vartheta(0) \vartheta_{2}(0) \vartheta_{3}(x) \vartheta_{3}(x)}{1-R^{2}(x)} \\
& =\frac{2 R}{1+R^{2}(x)} \cdot \frac{\sqrt{\left(1+\frac{i k^{\prime}}{k} R^{2}(x)\right)\left(1-\frac{i k}{k^{\prime}} R^{2}(x)\right)}}{1-\vartheta^{2}(x)}
\end{aligned}
$$

Real values for $R(x)$ along the real axis are no longer obtained if the Jacobian constants $q, k, k^{\prime}$ are real, but it will be now shown that if $k, k^{\prime}$ are conjugate imaginaries, there will be a line through the origin on which $R(x)$ is real. Place $k^{\prime} / k=e^{i \phi}$ so as to make $R(2 x)$ real when $R(x)$ is real. Then since

$$
i\left(k / k^{\prime}-k^{\prime} / k\right)=2 \sin \phi,
$$

the radical will vary with $\phi$ between the limits $1+R^{2}(x)$ and $1-R^{2}(x)$. Thus the limits for $|q(x)|$ are those given by the inequalities of case 2 .

The integrals

$$
K=\int_{0}^{1} \frac{d x}{\sqrt{\left(1-x^{2}\right)\left(1-k^{2} x^{2}\right)}}, \quad K^{\prime}=\int_{0}^{1} \frac{d x}{\sqrt{\left(1-x^{2}\right)\left(1-k^{\prime 2} x^{2}\right)}} .
$$

are conjugate imaginaries and the real part of $K^{\prime} / K$ is positive. ${ }^{*}$ Then $q=e^{-\pi K / K^{\prime}}$ is a complex imaginary with an absolute value less than 1 so that the usual series defining $\vartheta_{1}(x), \vartheta_{3}(x)$ have a significance. If

$$
\tau=i K^{\prime} / K \equiv e^{i \delta}
$$

we get by well-known formulas for transformation

* See for example Schwarz's Formeln und Lehrsätze zum Gebrauche der elliptischen Functionen, p. 32 . 


$$
\begin{array}{r}
\frac{1}{\sqrt{i} \vartheta_{1}(x, \tau)}=\frac{1}{i} \vartheta(x, \tau+1)=-\sqrt{i \tau^{\prime}} e^{-i \tau^{\prime} x^{2} / \pi} \vartheta_{1}\left(x \tau^{\prime},-\tau^{\prime}\right), \\
\vartheta_{3}(x, \tau)=\vartheta(x, \tau+1)=-\sqrt{i \tau^{\prime}} e^{-i \tau^{\prime} x^{2} / \pi} \vartheta_{2}\left(x \tau^{\prime},-\tau^{\prime}\right),
\end{array}
$$

in which

$$
\tau^{\prime}=\frac{1}{\tau+1}=\frac{1}{2}-\frac{i \sin \delta}{2(1+\cos \delta)}
$$

Since $\sin \delta$ is positive, $q^{\prime}=e^{-i \pi \tau^{\prime}}$ is a pure imaginary having a modulus less than 1 , and the ordinary series apply for $\vartheta_{i}\left(x \tau^{\prime},-\tau^{\prime}\right)(i=1,2)$ with $q^{\prime}$ in place of $q$. For real values of the argument $x \tau^{\prime}$ these series are real save for a factor $\sqrt[4]{q^{\prime}}$ common to both series. Hence the quotient

$$
R(x)=\frac{\vartheta_{1}(x, \tau)}{\sqrt{i} \vartheta_{3}(x, \tau)} \equiv \frac{\vartheta_{1}\left(x \tau^{\prime},-\tau^{\prime}\right)}{\vartheta_{2}\left(x \tau^{\prime},-\tau^{\prime}\right)}
$$

is real along the line through the origin on which $x \tau^{\prime}$ is real. Now the zeros of $\vartheta_{1}(u, q)$ and $\vartheta_{2}(u, q)$ for real values of $u$ and all values of $|q|<1$ alternate along the real axis, being situated at $u=n \pi$ and $u=(2 n+1) \pi / 2$. Case 2 can now be discussed exactly as case 3 with like conclusion.

In case 4 the relation

$$
R(x)=\frac{2 R\left(\frac{x}{2}\right)}{1+R^{2}\left(\frac{x}{2}\right)}
$$

makes $\left|R\left(x^{\prime}\right)\right|<1$ and necessitates also that $R\left(x^{\prime}\right)$ and $R\left(x^{\prime} / 2\right)$ shall be of like sign, and therefore also all terms of (71). The determination of $R(x)$ is unique when $R\left(x^{\prime}\right)$ is given and is realized through $\pm \tanh c x$. Case 5 is very similar to cases 2 and 3 and is realized through $\pm \tan c x$.

It is noteworthy that all the real solutions are analytic, being expressible in terms of $\vartheta$-functions or their degenerations.

From the real solutions of $(B)$ those of $(A)$ can be quickly obtained. We have only to effect one resolution of each solution of $(B)$ into real components satisfying $(A)$ and then to multiply these components by a common arbitrary factor of form (11), with or without change of sign of either component. For cases 1 and 3 a real resolution is afforded by (I) and (II), the characteristic constant $q$ in the $\vartheta$-functions and $g_{2}, g_{3}$ in the $\sigma$-functions being real. The real factor $e^{-2 \eta_{1} \omega_{1} x^{2}}$ can also be dropped, being of form (11). In case 2 the constant $q$ is unreal and the resolution offered in III or (72) is not real. But

and

$$
\phi(x)=\frac{\vartheta_{1}(c x, i q)}{\vartheta_{2}(0, i q)}, \quad \psi(x)=\frac{\vartheta_{2}(c x, i q)}{\vartheta_{2}(0, i q)} \quad \text { (c and } q \text { real) }
$$




$$
\phi(x)=\sqrt{\left(e_{2}-e_{3}\right)\left(e_{2}-e_{1}\right)} \sigma(c x), \quad \psi(x)=\sigma_{2}(c x)
$$

afford real resolutions, it being understood that the constants $g_{2}, g_{3}$ of these $\sigma$-functions are real with negative discriminant so that the quasi-periods $2 \omega_{1}, 2 \omega_{3}$ are conjugate imaginaries.

\section{Solutions FOR THE COMPLEX Plane}

Consider now equations $(A)$, supposing the functions which they contain to be analytic over the complex plane and hence to be entire functions. The form of $\phi(x), \psi(x)$ was ascertained in $\S 7$ when their systems of zeros are simply periodic. We need therefore only consider the doubly periodic case. Let $\alpha, \alpha^{\prime}$ be a primitive pair of zeros. We will take the positive real axis through the one or the other of these points, so making our choice that the other zero is made thereby to have a negative coefficient for its imaginary part. By replacing $x$ by $c x$ the real zero can then be made equal to $\pi$. If the other zero is denoted by $i \log q$, the absolute value of $q$ will be less than 1 . As shown in $\S 9$, the zeros are of first order. Form now in terms of $q$ the familiar infinite products for $\vartheta_{1}(x), \vartheta(x), \vartheta_{2}(x), \vartheta_{3}(x)$. Then we have in I-III three solutions $\phi_{i}(x), \psi_{i}(x)$ whose zeros occupy the only possible positions for the three allied solutions. Any other entire function whose zeros have the same positions as those of $\phi_{i}(x)$ or $\psi_{i}(x)$ can differ from it only by a factor of form $e^{G(x)}$, in which $G(x)$ denotes an entire function. Consider now any pair of functions $e^{G_{1}(x)} \phi_{i}(x), e^{G_{2}(x)} \psi_{i}(x)$, which give a solution of $(A)$. In accordance with (16) their quotient $R(x)$ must admit $2 \pi$ and $2 i \log q$ as periods. But $\phi_{i}(x) / \psi_{i}(x)$ by construction possesses these periods, and consequently $e^{G_{1}(x)-G_{2}(x)}$ must be doubly periodic. Being also an analytic function without infinities, this exponential must be a constant. Now any solution of $(B)$ ceases to be a solution when altered by a constant factor unless the factor is a fourth root of unity. Hence

$$
e^{G_{1}(x)}=\omega_{4} e^{G_{2}(x)},
$$

where $\omega_{4}$ is a fourth root of unity. Thus our pair of functions differ from $\phi_{i}(x), \psi_{i}(x)$, or an equivalent solution, only by a common factor which as such must be of form (11). Being also analytic, this factor is $e^{c x 2}$. Except therefore for such a common factor, the components of any analytic solution of $(A)$ with a doubly periodic system of zeros are expressible in one of the forms I-III in terms of $\vartheta$-functions (with $x$ replaced by $c x$ ), or as an equivalent solution.

Pass now to the consideration of analytic solutions of $(B)$. While to analytic solutions of $(A)$ there correspond analytic solutions of $(B)$, the converse is not evident without study. For the case of a doubly periodic system of zeros a comparison of any solution of $(B)$ with a corresponding quotient of $\vartheta$-func- 
tions possessing the same zeros and infinities does indeed serve to establish their identity save for a constant factor, but a corresponding basis for a like conclusion in the simply periodic case is not apparent. We shall proceed as follows so as to cover simultaneously both cases independently of any consideration of $(A)$.

An analytic solution of $(B)$ is determined by its section along a line through the origin. It has been seen that the latter is fixed by the values of $R(x)$ at two initial points $x^{\prime}$ and $2 x^{\prime}$ upon the line. A unique function is not thereby determined owing to the choice of roots offered for $R(x / 2)$ in (64), but the choice becomes definite when $x$ is sufficiently near to the origin. Consider now any particular analytic solution $R(x)$ and seek to determine a function $f(x)=\vartheta_{1}(c x, q) / \vartheta(c x, q)$ which will agree with $R(x)$ over a set of points $2 x^{\prime \prime}, x^{\prime \prime}, x^{\prime \prime} / 2, \cdots$ and therefore over the line on which they lie and over the entire plane.

First substitute the given initial values $R\left(2 x^{\prime}\right)$ and $R\left(x^{\prime}\right)$ in (70). The equation can then be solved for $k+1 / k$, giving two values of $k$ with product 1 . Take for $k$ one of the two values not exceeding 1 in absolute value. The parameter $q$ of the $\vartheta$-functions is expressible as an ordinary power series in $k$ whose coefficients are positive and have a sum equal to unity.* The series converges for $|k| \leqq 1$ and makes $|q| \leqq 1$. If $k=1$ or -1 , the functions $\tanh c x$ and $\tan c x$ respectively are to be substituted in place of $\vartheta_{1}(c x) / \vartheta(c x)$. Otherwise we have $|q|<1$ so that the ordinary series for $\vartheta_{1}(x)$ and $\vartheta(x)$ as functions of $x$ and $q$ apply. In this manner $q$ is determined so as to accord with the initial values.

When (70) is combined with (64) so as to eliminate $R(2 x)$, we obtain an equation between $R(x)$ and $R(x / 2)$ exactly like (70), except possibly for the sign of the radical, and containing the same value of $k+1 / k$. The value of $q$ continues therefore to correspond when $x^{\prime}$ is replaced by $x^{\prime} / 2^{m}$. Consider now the sequence

$$
R\left(2 x^{\prime}\right), \quad R\left(x^{\prime}\right), \quad R\left(x^{\prime} / 2\right), \quad \cdots .
$$

As already explained, each term after the first two is uniquely derivable by (64) from the two preceding, provided that $x^{\prime}$ is selected sufficiently near to the origin, as will be supposed. A like remark applies to a similar sequence formed for the solution $f(x)=\vartheta_{1}(x) / \vartheta(x)$. Now a sufficiently small vicinity of $x=0$ will be built by $f(x)$, or the two above degenerate functions, with continuous 1-1 correspondence of points upon a vicinity of the origin of the $f$-plane. If $m$ is sufficiently large, $R\left(x^{\prime} / 2^{m}\right)$ must fall in this vicinity and hence there is a point $x^{\prime \prime}$ such that $f\left(x^{\prime \prime}\right)=R\left(x^{\prime} / 2^{m}\right)$. By a proper substitution of $c x$ for $x$ in $f(x)$ the point $x^{\prime \prime}$ may be made to coincide with

* Cf. Schwarz, loc. cit., p. 54. 
$x^{\prime} / 2^{m}$. If furthermore $m$ is sufficiently large, $x^{\prime \prime}$ will lie so close to the origin that $f\left(2 x^{\prime \prime}\right) / f\left(x^{\prime \prime}\right)$ and $R\left(2 x^{\prime \prime}\right) / R\left(x^{\prime \prime}\right)$ in accordance with (59) will both be very nearly equal to 2 . Since also $f\left(2 x^{\prime \prime}\right)$ and $R\left(2 x^{\prime \prime}\right)$ can both be obtained from $f\left(x^{\prime \prime}\right)=R\left(x^{\prime \prime}\right)$ by the same equation (70), we must have $f\left(2 x^{\prime \prime}\right)=R\left(2 x^{\prime \prime}\right)$. Agreeing then at the two points $x^{\prime \prime}, 2 x^{\prime \prime}$ the two functions must also agree over the set $x^{\prime \prime} / 2, x^{\prime \prime} / 4, \cdots$ and hence over the line through the origin containing these points and over the complex plane. Thus any analytic solution of $(B)$ is found to be identical with some function

$$
\vartheta_{1}(c x, q) / \vartheta(c x, q)
$$

or with one of its degenerations.

In conclusion, we will make a few incomplete remarks regarding the solution of $(B)$ when $R(x)$ is not analytic but merely fulfills the continuity assumptions of $\S 10$. The solutions depend upon three parameters, which we assume to be the values of $R(x)$ at three initial points $x^{\prime}, y^{\prime}, x^{\prime}-y^{\prime}$ which do not lie on a common line through the origin. Assume arbitrarily these values, avoiding for convenience the values $0, \pm 1, \pm i, \infty$. Then by use of $(B)$ the function $R(x)$ may be determined over the dense network

$$
\pm \frac{n x^{\prime}}{2^{m}} \pm \frac{n y^{\prime}}{2^{m \prime}} \quad\left(m, n, m^{\prime}, n^{\prime}=0,1,2, \cdots\right) .
$$

It does not, however, appear under what conditions the various sets of values thus obtained are consistent with the requirement of continuity. For a given solution its determination by specification of its values at three initial points will be unique, provided these values are sufficiently near to the origin. From a variety of loose considerations it seems altogether likely that all non-analytic solutions, so far as they do not fall under the somewhat trivial case of $\S 3$, are merely affine distortions of analytic solutions, exactly as established in the simply periodic case. If, however, this is not the case, then there are other non-analytic solutions obeying the same general laws as the $\vartheta$-functions.

UNIVERSITY OF WISCONSIN,

July, 1915 